\title{
EPR argument and Bell inequalities for Bose-Einstein spin condensates
}

\author{
F. Laloë (a) and W. J. Mullin (b) \\ (a) Laboratoire Kastler Brossel, ENS, UPMC, CNRS; 24 rue Lhomond, 75005 Paris, France \\ (b) Department of Physics, University of Massachusetts, Amherst, Massachusetts 01003 USA
}

November 11, 2018

\begin{abstract}
We discuss the properties of two Bose-Einstein condensates in different spin states, represented quantum mechanically by a double Fock state. Individual measurements of the spins of the particles are performed in transverse directions (perpendicular to the spin quantization axis), giving access to the relative phase of the two macroscopically occupied states. Before the first spin measurement, the phase is completely undetermined; after a few measurements, a more and more precise knowledge of its value emerges under the effect of the quantum measurement process. This naturally leads to the usual notion of a quasi-classical phase (Anderson phase) and to an interesting transposition of the EPR (Einstein-Podolsky-Rosen) argument to macroscopic physical quantities. The purpose of this article is to discuss this transposition, as well as situations where the notion of a quasi-classical phase is no longer sufficient to account for the quantum results, and where significant violations of Bell type inequalities are predicted.

Quantum mechanically, the problem can be treated exactly: the probability for all sequences of results can be expressed in the form of a double integral, depending on all parameters that define the experiment (number of particles, number and angles of measurements). We discuss the differences between this case and the usual two-spin case. We then discuss the effect of the many parameters that the experimenters can adjust for their measurements, starting with a discussion of the effect of the angles of measurement (the "settings"), and then envisaging various choices of the functions that are used to obtain violation of BCHSH inequalities. We then discuss how the "sample bias loophole" (often also called "efficiency loophole") can be closed in this case, by introducing a preliminary sequence of measurements to localize the particles into "measurement boxes". We finally show how that the same non-local effects can be observed with distinguishable spins.
\end{abstract}

\section{Introduction}

Two of Einstein's many famous contributions to physics are the theoretical discovery in 1925 of the Bose-Einstein condensation in a gas [1] and, ten years later, the celebrated Einstein-Podolsky-Rosen (EPR) argument [2]. Both have had an enormous influence in the discipline and stimulated much work, both theoretical and experimental. Although they are both fundamental, these contributions appear almost completely disconnected: the former is more "standard" physics, initiating the domain of quantum statistical physics, with many applications in gas and condensed matter physics; the latter belongs to the foundations of quantum mechanics and has indeed attracted the attention of philosophers. It is therefore interesting to realize that both contributions are connected logically, and that the study of interfering Bose-Einstein condensates may shed a new light on the fundamental debate initiated by EPR. The basic reason is that, while the original EPR argument applies to two microscopic particles, its transposition to Bose-Einstein condensates introduces systems that can be macroscopic. The "elements 
of reality", introduced by EPR as attached to microscopic particles, then characterize the relative phase between the two condensates which, in the case of spin condensates, may determine a macroscopic spin orientation. This is an important difference: while one can argue as Bohr 3 that elements of reality for microscopic particles do not exist independently of the measurement apparatuses, it is more difficult to deny that macroscopic physical systems possess an independent physical reality. As a consequence, the EPR argument then becomes more compelling 4. Another interesting feature is that, while in the original example the EPR element of reality (and therefore an additional, or "hidden" variable) appears as completely foreign to standard quantum mechanics, with condensates the relative phase emerges rather naturally within its formalism of standard quantum mechanics, simply as a consequence of particle number conservation 4 .

A natural question then is whether condensates can lead to violations of local realism, as systems of two particles do, in other words whether violations of Bell-type inequalities [5, 6] also occur with macroscopic condensates. In view of this macroscopic character, one could expect that the answer to this question is no. Actually, it turns out that it is yes: as shown in a letter [7 recently, strong violations do occur when measuring the individual transverse spin orientation of particles in a double spin condensate with equal populations, even for arbitrarily large condensates. An essential condition, nevertheless, is that the spins of all particles be measured; missing one or more spins makes the violation disappear. The relative phase that emerges under the effect of the first few quantum measurements behaves like a quasi-classical variable, so that violations are impossible in this regime; nevertheless, if one continues the sequence of measurements until its maximum, one reaches situations that can no longer be understood with this classical phase, but recover an intrinsic quantum character.

The purpose of the present article is to discuss in more detail several aspects of the questions treated in 7 as well as those that were not be treated there. In $\S$ 2, we define the physical system under consideration and recall the notation, as well as previous results. In $\S 3$ we come back to the EPR argument in the context of the relative phase of two condensates, and in $\S$ to violations of Bell inequalities obtained within stochastic local realist theories (as opposed to the usual two-spin case where deterministic local realist theories are more natural). Generally speaking, a useful feature of transverse spin measurements in condensates is that one can calculate exactly the effect of many parameters defining the measurements: angles at which the spins are measured, number of particles and number of measurements, various functions used to obtain violations of the inequalities, etc.; the discussion of the effect of these parameters is

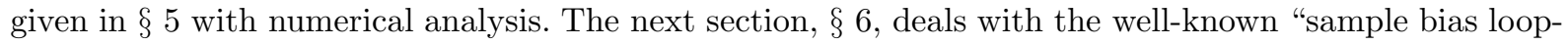
hole" (often also called "detection, or efficiency, loophole"), which can be closed (in thought experiments) with the help of preliminary measurements, exactly in the perspective proposed by Bell 8 , to handle such situations. In $\S \mathbf{7}$ we show that the violations of local realism are not related to boson statistics, but that the same violations can be obtained with distinguishable spins, provided they are in an appropriate initial state. We then draw conclusions in $\S 8$.

\section{Summary of previous results}

We recall in this section the results already obtained in [4, 7, starting with the equations that show how a phase naturally emerges from the predictions of standard quantum mechanics in a series of transverse spin measurements. We then discuss in what conditions this phase behaves as a quasi-classical quantity, or retains a strong quantum character; in the latter case, we briefly introduce the violation of Bell inequalities and local realism that can be obtained, keeping a more detailed discussion for $\S \S$ and 5 ,

\subsection{Quantum predictions}

Consider an ensemble of $N_{+}$particles in a state characterized by an orbital state $u(\mathbf{r})$ and a spin state + , and $N_{-}$particles in the same orbital state $v(\mathbf{r})$ with spin orientation -. This physical system is described 
quantum mechanically by a double Fock state:

$$
\left|\Phi>\sim\left[\left(a_{u,+}\right)^{\dagger}\right]^{N_{+}}\left[\left(a_{v,-}\right)^{\dagger}\right]^{N_{-}}\right| \text {vac. }>
$$

where $a_{u,+}$ and $a_{u,-}$ are the destruction operators associated with the two populated single-particle states and $\mid$ vac. $>$ is the vacuum state. The total number of particles is:

$$
N=N_{+}+N_{-}
$$

The operators associated with the local density and the local density of spins can be expressed as function of the two fields operators $\Psi_{ \pm}(\mathbf{r})$ associated with the two internal states \pm as:

$$
\begin{aligned}
& n(\mathbf{r})=\Psi_{+}^{\dagger}(\mathbf{r}) \Psi_{+}(\mathbf{r})+\Psi_{-}^{\dagger}(\mathbf{r}) \Psi_{-}(\mathbf{r}) \\
& \sigma_{z}(\mathbf{r})=\Psi_{+}^{\dagger}(\mathbf{r}) \Psi_{+}(\mathbf{r})-\Psi_{-}^{\dagger}(\mathbf{r}) \Psi_{-}(\mathbf{r}) \\
& \sigma_{x}(\mathbf{r})=\Psi_{+}^{\dagger}(\mathbf{r}) \Psi_{-}(\mathbf{r})+\Psi_{-}^{\dagger}(\mathbf{r}) \Psi_{+}(\mathbf{r}) \\
& \sigma_{y}(\mathbf{r})=i\left[\Psi_{-}^{\dagger}(\mathbf{r}) \Psi_{+}(\mathbf{r})-\Psi_{+}^{\dagger}(\mathbf{r}) \Psi_{-}(\mathbf{r})\right]
\end{aligned}
$$

while the spin component in the direction of plane $x O y$ making an angle $\varphi$ with $O x$ is:

$$
\sigma_{\varphi}(\mathbf{r})=e^{-i \varphi} \Psi_{+}^{\dagger}(\mathbf{r}) \Psi_{-}(\mathbf{r})+e^{i \varphi} \Psi_{-}^{\dagger}(\mathbf{r}) \Psi_{+}(\mathbf{r})
$$

When a measurement of this component performed at point $\mathbf{r}$ provides the result $\eta= \pm 1$, the corresponding projector is:

$$
P_{\eta= \pm 1}(\mathbf{r}, \varphi)=\frac{1}{2}\left[n(\mathbf{r})+\eta \sigma_{\varphi}(\mathbf{r})\right]
$$

For a series of $N$ measurements that are performed at different points $\mathbf{r}_{i}$ (ensuring that the projectors all commute) along directions $\varphi_{i}$, the probability for obtaining a series of results $\eta_{i} \pm 1$ can be written as:

$$
\mathcal{P}\left(\eta_{1}, \eta_{2}, \ldots \eta_{N}\right)=<\Phi\left|P_{\eta_{1}}\left(\mathbf{r}_{1}, \varphi_{1}\right) \times P_{\eta_{2}}\left(\mathbf{r}_{2}, \varphi_{2}\right) \times \ldots . P_{\eta_{N}}\left(\mathbf{r}_{N}, \varphi_{N}\right)\right| \Phi>
$$

Strictly speaking, this expression is not a probability but a density of probability, which must be integrated in a finite volume $\Delta_{\mathbf{r}}$ to provide a probability; for instance, the probability for finding a particle in volume $\Delta_{\mathrm{r}}$ is:

$$
P\left(\Delta_{\mathbf{r}}\right)=\int_{\Delta_{\mathbf{r}}} d^{3} r^{\prime} n\left(\mathbf{r}^{\prime}\right)=\int_{\Delta_{\mathbf{r}}} d^{3} r^{\prime}\left[\Psi_{+}^{\dagger}\left(\mathbf{r}^{\prime}\right) \Psi_{+}\left(\mathbf{r}^{\prime}\right)+\Psi_{-}^{\dagger}\left(\mathbf{r}^{\prime}\right) \Psi_{-}\left(\mathbf{r}^{\prime}\right)\right]
$$

A similar expressions for finding its spin along any direction $\phi$ is obtained by integrating (5) over space. Here, for convenience we do not write the integrals explicitly, but it must be understood that expression (6) as well as those we write below are in fact integrated over all position variables $\mathbf{r}_{1}, \mathbf{r}_{2}$, etc. in spatially disconnected 1 volumes $\Delta_{1}, \Delta_{2}$, etc. As in the second ref [4], we call $\Delta_{1}, \Delta_{2}, .$. the "detection boxes" and refer the reader to this reference for a more detailed discussion, including the case where no particle at all is detected in the detection box; we also come back to this point in $\S 6$,

As in ref. [7] we now substitute the expression for $\sigma_{\varphi}(\mathbf{r})$ into (5) and (6). In the product of projectors appearing in (6), all $\mathbf{r}$ 's are different and commutation allows us to push all the field operators to the right, all their conjugates to the left; then that each $\Psi_{+}(\mathbf{r})$ acting on $\mid \Phi>$ can be replaced by $u(\mathbf{r}) \times a_{u,+}$, each $\Psi_{-}(\mathbf{r})$ by $v(\mathbf{r}) \times a_{u,-}$, and similarly for the Hermitian conjugates. With our initial state, a non-zero result can be obtained only if exactly $N_{+}$operators $a_{u,+}$ appear in the term considered,

\footnotetext{
${ }^{1}$ If there was an overlap between the "detection boxes", the projectors would no longer commute and equation (6) would no longer be valid; it would then be necessary to use the complete "Wigner formula" with twice as many projectors ordered in a symmetric way.
} 
and $N_{-}$operators $a_{v,-}$; a similar condition exists for the Hermitian conjugate operators. To express these conditions, we introduce two additional variables. The first variable $\lambda$ ensures an equal number of creation and destruction operators in the internal states \pm ; each $\Psi_{+}\left(\right.$or $\left.a_{u,+}\right)$ is multiplied by $e^{i \lambda}$, and each $\Psi_{+}^{\dagger}\left(\right.$ or $\left.a_{u,+}^{\dagger}\right)$ by $e^{-i \lambda}$ (operators related to the - spin state remain unchanged), and the conservation of the number of particles in spin + states is then obtained by the following integration:

$$
\int_{-\pi}^{\pi} \frac{d \lambda}{2 \pi} e^{i n \lambda}=\delta_{n, 0}
$$

(since the total number of particles is unchanged, the number of - particles is then also automatically conserved). The second variable $\Lambda$ expresses that the difference between the number of destruction operators in states + and - is exactly $N_{+}-N_{-}$, through the integral:

$$
\int_{-\pi}^{\pi} \frac{d \Lambda}{2 \pi} e^{-i n \Lambda} e^{i\left(N_{+}-N_{-}\right) \Lambda}=\delta_{n, N_{+}-N_{-}}
$$

This time, each $\Psi_{+}$( or $\left.a_{u,+}\right)$ is multiplied by $e^{-i \Lambda}$ and each $\Psi_{-}$(or $a_{v,-}$ ) by $e^{i \Lambda}$ (but creation operators remain unchanged). The introduction of all these exponentials into the product of projectors (5) in (6) then provides the expression (c.c. means complex conjugate):

$$
N_{+} ! N_{-} ! \prod_{j=1}^{N} \frac{1}{2}\left[e^{-i \Lambda}\left|u\left(\mathbf{r}_{j}\right)\right|^{2}+e^{i \Lambda}\left|v\left(\mathbf{r}_{j}\right)\right|^{2}+\eta_{j}\left(u^{*}\left(\mathbf{r}_{j}\right) v\left(\mathbf{r}_{j}\right) e^{i\left(\lambda-\varphi_{j}+\Lambda\right)}+\text { c.c. }\right)\right]
$$

where the field operators are replaced by a constant number $N_{+} ! N_{-}$! because, after integration over $\lambda$ and $\Lambda$, the only surviving terms are all associated with the same matrix element, that of the product of $N_{+}$operators $a_{u,+}^{\dagger}$ and $N_{-}$operators $a_{u,-}^{\dagger}$ followed by the same sequence of destruction operators inside state $\mid \Phi>$. To simplify the equations, from now on we assume that all measurements are made at points where the two wave functions $u(\mathbf{r})$ and $u(\mathbf{r})$ are equal2; we can then write the probability as:

$$
\mathcal{P}\left(\eta_{1}, \eta_{2}, \ldots \eta_{N}\right) \sim \int_{-\pi}^{\pi} \frac{d \lambda}{2 \pi} \int_{-\pi}^{+\pi} \frac{d \Lambda}{2 \pi} e^{i\left(N_{+}-N_{-}\right) \Lambda} \prod_{j=1}^{N}\left\{\left|u\left(\mathbf{r}_{j}\right)\right|^{2} \frac{1}{2}\left[e^{i \Lambda}+e^{-i \Lambda}+\eta_{j}\left(e^{i\left(\lambda-\varphi_{j}+\Lambda\right)}+\text { c.c. }\right)\right]\right\}
$$

By changing one integration variable $\left(\lambda^{\prime}=\lambda+\Lambda\right)$ and using $\Lambda$ parity, we obtain:

$$
\mathcal{P}\left(\eta_{1}, \eta_{2}, \ldots \eta_{N}\right)=\frac{1}{2^{N} C_{N}} \int_{-\pi}^{+\pi} \frac{d \Lambda}{2 \pi} \cos \left[\left(N_{+}-N_{-}\right) \Lambda\right] \int_{-\pi}^{+\pi} \frac{d \lambda^{\prime}}{2 \pi} \prod_{j=1}^{N}\left[\cos (\Lambda)+\eta_{j} \cos \left(\lambda^{\prime}-\varphi_{j}\right)\right]
$$

where the normalization coefficient $C_{N}$ is obtained by writing that the sum of probabilities of all possible sequences of $\eta$ 's is 1 (we come back to this point in $\S[$ ):

$$
C_{N}=\int_{-\pi}^{+\pi} \frac{d \Lambda}{2 \pi} \cos \left[\left(N_{+}-N_{-}\right) \Lambda\right][\cos (\Lambda)]^{N}
$$

In the above equations, it is sometimes convenient to reduce the integration domain of $\Lambda$ to the interval between $-\pi / 2$ and $+\pi / 2$. This is possible, since (before variable $\lambda$ is changed into $\lambda^{\prime}$ ) changing $\Lambda$ into $\pi-\Lambda$ introduces into (12) a factor $(-1)^{N_{+}-N_{-}+N}$, or $(-1)^{2 N_{+}}$, which is 1 .

\footnotetext{
${ }^{2}$ If they have equal modulus but different phases, their phase difference at point $\mathbf{r}_{j}$ is simply added to the measurement angle $\varphi_{j}$.
} 
Let us assume unequal populations, for instance $N_{+}>N_{-}$. Then, in the product over $j$ contained in (12), only some terms provide a non-zero contribution in the integral over $\Lambda$; in fact, we must choose at least $N_{+}-N_{-}$factors contributing through $\cos (\Lambda)$, and therefore at most $N-\left(N_{+}-N_{-}\right)=2 N_{-}$ factors contributing through the $\eta_{j}$ and $\phi_{j}$ dependent terms. We see that $2 N_{-}$is the maximum number of spins that can provide a transverse spin measurement that depends on the result $\eta$ and on the angle of measurement; all the others have equal probabilities $1 / 2$, whatever the angle of measurement is. This is physically understandable, since $\left(N_{+}-N_{-}\right)$spins + are unmatched with - spins, and can thus not be found in a coherent superposition of the two spin states; they then provide $1 / 2$ probabilities for any direction of transverse spin measurement. We therefore see that all spins can contribute coherently to the measurement only if $N_{+}=N_{-}$.

The above equations are valid only when the number of measurements $M$ is equal to $N$, but can still be used when it is smaller. The reason is that any sequence of $M<N$ measurements can always be completed by additional $N-M$ measurements, leading to probability (12). We can therefore take the sum of (12) over the results of the missing $N-M$ measurements, which amounts to setting the corresponding $\eta_{j}$ equal to zero and doubling the remaining term. We then obtain:

$$
\mathcal{P}\left(\eta_{1}, \eta_{2}, \ldots \eta_{M}\right)=\frac{1}{2^{M} C_{N}} \int_{-\pi}^{+\pi} \frac{d \Lambda}{2 \pi} \cos \left[\left(N_{+}-N_{-}\right) \Lambda\right][\cos \Lambda]^{N-M} \int_{-\pi}^{+\pi} \frac{d \lambda}{2 \pi} \prod_{j=1}^{M}\left[\cos (\Lambda)+\eta_{j} \cos \left(\lambda-\varphi_{j}\right)\right]
$$

Finally, what we will need below is the value of the quantum average of the product of results, i.e., the sum $\mathcal{P}\left(\eta_{1}, \eta_{2}, \ldots \eta_{M}\right)$ times this product over all possible values of the $\eta$ 's. This sum, according to (12), is given by:

$$
E\left(\varphi_{1}, \varphi_{2}, \ldots \varphi_{M}\right)=\left(C_{N}\right)^{-1} \int_{-\pi}^{+\pi} \frac{d \Lambda}{2 \pi} \cos \left[\left(N_{+}-N_{-}\right) \Lambda\right][\cos \Lambda]^{N-M} \int_{-\pi}^{+\pi} \frac{d \lambda}{2 \pi} \prod_{j=1}^{M} \cos \left(\lambda-\varphi_{j}\right)
$$

\subsection{Classical or quantum regime of the phase}

The relative phase $\lambda$ is a priori completely absent from Fock states in standard quantum mechanics, but nevertheless appears naturally in its formalism as a mere consequence of the conservation of the number of particles3. The phase actually occurs in an integral; for the first measurement, the integral merely expresses that the phase is initially completely undetermined, as one could expect; for a series of measurements, the phase integral provides the relation between the successive results and introduces their correlations. Depending on the measurements, this phase takes a classical or a quantum character.

If the number of experiments $M$ is much less than a very large $N$, and if $N_{+}=N_{-}$, because $\cos \Lambda^{N-M}$ peaks up sharply at $\Lambda=0$, equation (14) becomes:

$$
\mathcal{P}\left(\eta_{1}, \eta_{2}, \ldots \eta_{M}\right) \simeq \frac{1}{2^{M}} \int_{-\pi}^{+\pi} \frac{d \lambda}{2 \pi} \prod_{j=1}^{M}\left[1+\eta_{j} \cos \left(\lambda-\varphi_{j}\right)\right]
$$

We then recover the results of [4] as well as of previous work (refs [9] to [18]). For a given $\lambda$, the probabilities can be obtained by considering that the sample is completely polarized in a transverse direction determined by angle $\lambda$; the spin measurements then become independent processes with individual probabilities given by $\left\{1+\eta_{j} \cos \left(\lambda-\varphi_{j}\right)\right\}$, exactly as for a single isolated spin; the additional ingredient is the $\lambda$ integral, which expresses that all values of $\lambda$ are equally probable and introduces the correlations.

\footnotetext{
${ }^{3}$ More precisely, the conservation of the difference of the number of particles in the two internal states, which is the conjugate variable of the relative phase.

${ }^{4}$ Here we take the point of view where the $\Lambda$ integration domain is between $-\pi / 2$ and $+\pi / 2$; otherwise, we should also take into account a peak around $\Lambda=\pi$.
} 
In this case, all predictions of quantum mechanics lead to predictions that are perfectly compatible with the idea of a pre-existing phase, which takes a well-defined value before any measurement and remains constant5; this value is initially completely unknown and changes from one realization of the experiment to the next. This fits well with the concept of the Anderson phase, which originates from spontaneous symmetry breaking at the phase transition (Bose-Einstein condensation): at this transition point, the quantum system chooses a phase, which takes a completely random value, but then plays the role of a classical variable in the limit of very large systems.

On the other hand, if $N-M$ is not a large number, the peaking effect of $\cos \Lambda^{N-M}$ does not occur anymore and $\Lambda$ can take values close to $\pi / 2$, so that the terms in the product inside the integral are no longer necessarily positive; an interpretation in terms of probabilities then becomes impossible - see $\S$ 4.2 for more details. In these cases, the phase does not behave as a semi-classical variable, but retains a strong quantum character; the variable $\Lambda$ controls the amount of quantum effects.

\subsection{Violations of local realism in the quantum regime}

Indeed, in the quantum regime where $\Lambda$ is not limited to values around zero, equations (14) and (15) may contain strong violations of Bell inequalities and therefore of local realism. Consider a thought experiment with two condensates, each in a different spin state (two eigenstates of the spin component along the quantization axis $O z$ ). The two condensates extend into two remote regions of space $D_{A}$ and $D_{B}$ where they overlap and have equal orbital wave functions, and where two experimentalists Alice and Bob measure the spins of the particles in arbitrary transverse directions (any direction perpendicular to $O z$ ), see Fig. 1. We assume that all measurements performed by Alice are made along the same direction $\varphi_{a}$, which plays here the usual role of the "setting" $a$, while all measurements performed by Bob are made with another single angle $\varphi_{b}$. To complete the analogy with the usual situation with two particles, we assume that Alice retains of all her measurements just their product $\mathcal{A}$, while Bob retains only the product $\mathcal{B}$ of his results (these numbers are both \pm 1 , the parities resulting of all the local measurements) - other possibilities than these simple products are considered in $\S 5$.

We now assume that Alice, in successive realizations of the experiment, uses two possible orientations $\varphi_{a}$ and $\varphi_{a}^{\prime}$, and that Bob does the same with two possible orientations $\varphi_{b}$ and $\varphi_{b}^{\prime}$. Within local realism, for each realization of the experiment, it is possible to define two numbers $\mathcal{A}$, $\mathcal{A}^{\prime}$, both equal to \pm 1 , and associated with the two possible products of results that Alice will observe, depending of her choice of orientation; the same is obviously true for Bob, introducing $\mathcal{B}$ and $\mathcal{B}^{\prime}$. Since $\mathcal{A B}= \pm \mathcal{A}^{\prime} \mathcal{B}^{\prime}$, either $\mathcal{A B}+\mathcal{A B}^{\prime}$ or $\mathcal{A}^{\prime} \mathcal{B}-\mathcal{A}^{\prime} \mathcal{B}^{\prime}$ vanishes, and the following inequality is straightforward:

$$
-2 \leq \mathcal{A B}+\mathcal{A B}^{\prime} \pm\left(\mathcal{A}^{\prime} \mathcal{B}-\mathcal{A}^{\prime} \mathcal{B}^{\prime}\right) \leq 2
$$

As a consequence, the average of the products in (17), obtained by repeating the experiment and the measurements many times, must be between -2 and +2 ; this is the famous BCHSH (Bell, Clauser, Horne, Shimony and Holt) inequality [19, 20, a consequence of local realism.

Within standard quantum mechanics, the above reasoning no longer holds. As emphasized by Peres [21, "unperformed experiments have no results", so that several of the numbers appearing in (17) are undefined; in fact, only two of them are defined after the experiment has been performed with a given choice of the orientations. Consequently, while one can calculate from (15) the quantum average $\langle Q\rangle$ of the sum of products of results appearing in the middle of (17), there is no special reason why $\langle Q\rangle$ should be limited between +2 and -2 . Situations where the inequality:

$$
-2 \leq\langle Q\rangle \leq 2
$$

\footnotetext{
${ }^{5}$ In our calculations, we have assumed that the measurements are all made in a very short period of time, so that the evolution of the system between them can be ignored.

${ }^{6} \mathrm{~A}$ more precise statement would be: "for a given realization of the experiments, unperformed experiments have no results; or, if they do, each result depends not only on the local setting but also (and non-locally) on the other remote setting".
} 

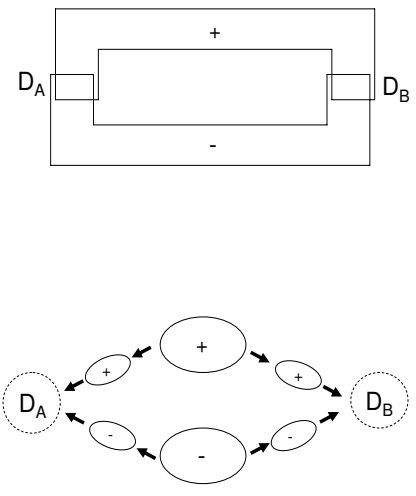

Figure 1: Two condensates with fixed number of particles, one having up spins and the other down spins, overlap in two remote regions of space $D_{A}$ and $D_{B}$ where measurements of transverse spin orientations are made by Alice and Bob. In the upper part of the figure, we assume that each of the orbital wave functions associated with the two condensates are simply connected, and extend continuously between the two remote measurement sites. This is not a necessary condition, nevertheless. For instance, as shown schematically in the lower part of the figure, we can assume that the wave function of each condensate is coherently split into two parts and separates into two disconnected components (each of which will then contain a fluctuating number of particles), and that each of which propagates to one measurement region separately.

is violated are sometimes called "quantum non-local situations".

Situation in which (16) holds can not lead to such violations, since this equation contains positive probabilities inside the integral and has precisely the form from which Bell inequalities can be derived (we come back to this point in more detail in $\S$ 4.1). So, here we no longer assume that $M \ll N$ but consider the other extreme, $M=N$. The simplest case occurs when $N_{+}=N_{-}=1$ and when Alice and Bob make one measurement each; it is then easy to see that (11) defines a triplet spin state $\mid S=1, M_{S}=0>$. In this case, it is well-known 7 that a violation of (18) occurs, by a factor $\sqrt{2}$ when the angles form a "fan" 8 spaced by $\chi=\pi / 4$; this saturates the Cirel'son bound 22 . But the violations also occur for arbitrarily large values of $N_{+}$and $N_{-}$: for instance, consider the case $M=N, N_{a}=1$ (Alice makes one measurement only) and $N_{b}=N-1$ (Bob makes the maximum number of remaining possible measurements). Then

\footnotetext{
${ }^{7}$ When one measures the components of the spins along directions that are perpendicular to the quantization axis, the predictions of quantum mechanics are the same for this triplet case and the singlet state $\mid S=0, M_{S}=0>$, provided one just reverses the direction of one measurement.

${ }^{8}$ The term "fan" refers to the angles arranged as $\varphi_{a b}=\varphi_{b a^{\prime}}=\varphi_{b^{\prime} a}=\chi$ and $\varphi_{b^{\prime} a^{\prime}}=3 \chi$ where $\varphi_{a b} \equiv \varphi_{a}-\varphi_{b}$.
} 
equation (15) becomes:

$$
\begin{aligned}
& E\left(\varphi_{a}, \varphi_{b}\right)=\int_{-\pi}^{\pi} \frac{d \lambda}{2 \pi}\left[\cos ^{1}\left(\lambda-\varphi_{a}\right) \cos ^{N-1}\left(\lambda-\varphi_{a}\right)\right]\left[\int_{-\pi}^{\pi} \frac{d \lambda}{2 \pi} \cos ^{N} \lambda\right]^{-1} \\
& =\int_{-\pi}^{\pi} \frac{d \lambda^{\prime}}{2 \pi}\left[\cos ^{1}\left(\lambda^{\prime}-\varphi_{a}+\varphi_{b}\right) \cos ^{N-1} \lambda^{\prime}\right]\left[\int_{-\pi}^{\pi} \frac{d \lambda}{2 \pi} \cos ^{N} \lambda\right]^{-1} \\
& =\int_{-\pi}^{\pi} \frac{d \lambda^{\prime}}{2 \pi}\left[\left(\cos \lambda^{\prime} \cos \left(\varphi_{a}-\varphi_{b}\right) \cos ^{N-1} \lambda^{\prime}\right]\left[\int_{-\pi}^{\pi} \frac{d \lambda}{2 \pi} \cos ^{N} \lambda\right]^{-1}=\cos \left(\varphi_{a}-\varphi_{b}\right)\right.
\end{aligned}
$$

This result is precisely the same as that for the two-particle case, so that the Cirel'son bound is saturated again. For other values of $N_{a}$ and $N_{b}$, substantial violations of (18) continue to occur 77; we refer to $\S$ (5 for more details.

\section{A macroscopic element of reality}

We begin this section with a brief discussion of the Leggett-Sols argument which, from a different point of view, leads to the same conclusions as the EPR argument; we then discuss in more detail the transposition of the EPR argument to spin condensates.

\subsection{The Leggett-Sols argument}

Leggett and Sols 23] discuss a situation that has strong similarities with double spin condensates: two superconductors, initially in Fock (number) states, are coupled by a Josephson junction, which creates a current flow between them; the phase of this time oscillating current corresponds to the relative phase of the two superconductors. In standard quantum mechanics, initially this phase is completely undetermined but, as soon as the time dependence of the current is measured, the phase is created by the very act of measurement; it takes some random, but well-defined, value. The authors ask "Does the act of looking to see whether a Josephson current flows force the system into an eigenstate of current, and hence of relative phase?". The answer of standard quantum mechanics to this question is "yes", but the authors point out that "if one thinks about it seriously, this answer is bizarre in the extreme". To illustrate why, they suppose that the current is of order of, say, kiloamps, and that it is measured through the observation of a small magnet needle. They then ask: "Can it really be that by placing a minuscule compass needle next to the system, with a weak light beam to read off its position, we can force the system to realize a definite macroscopic value of the current? Common sense certainly rebels against this conclusion, and we believe that in this case common sense is right". In other words, because the current is arbitrarily large, its phase can not be created by a tiny measurement apparatus; it must already have existed before the measurement. Since this "element of reality" is not contained in standard quantum mechanics, this theory is incomplete. Here the argument is not locality, as in the EPR argument, but simply that a very small system can not completely modify an arbitrarily large system through a quasi instantaneous and mysterious measurement process, without any precise physical mechanism to explain why and how.

Double condensates undergoing transverse spin measurements, with a number of measurements $M \ll$ $N$, are very similar to the case discussed by Leggett and Sols. From (16), we can obtain that the probability of finding $\eta_{M}$ in the $M$-th measurement along $\varphi_{k}$, having found $\eta_{1}, \cdots \eta_{M-1}$ in the previous measurements along $\varphi_{1}, \cdots \varphi_{M-1}$ :

$$
\mathcal{P}\left(\eta_{M}\right)=\frac{1}{2^{M}} \int_{-\pi}^{+\pi} \frac{d \lambda}{2 \pi}\left\{1+\eta_{M} \cos \left(\lambda-\varphi_{k}\right)\right\} g_{M}(\lambda)
$$

where:

$$
g_{M}(\lambda)=\prod_{j=1}^{M-1}\left\{1+\eta_{j} \cos \left(\lambda-\varphi_{j}\right)\right\}
$$


(these equations are valid only if $M \ll N)$. The evolution of this "probability function" was studied in ref. 25] where it was shown that it peaks up sharply after only a few measurements. A typical result is shown in Fig. 2 where the measurements are done at a single angle $\varphi_{a}=0$. Two peaks arise because, with a single angle of measurement, the sign of the relative phase is not determined, but a very small number of additional measurements at a new angle causes the collapse to a single peak.

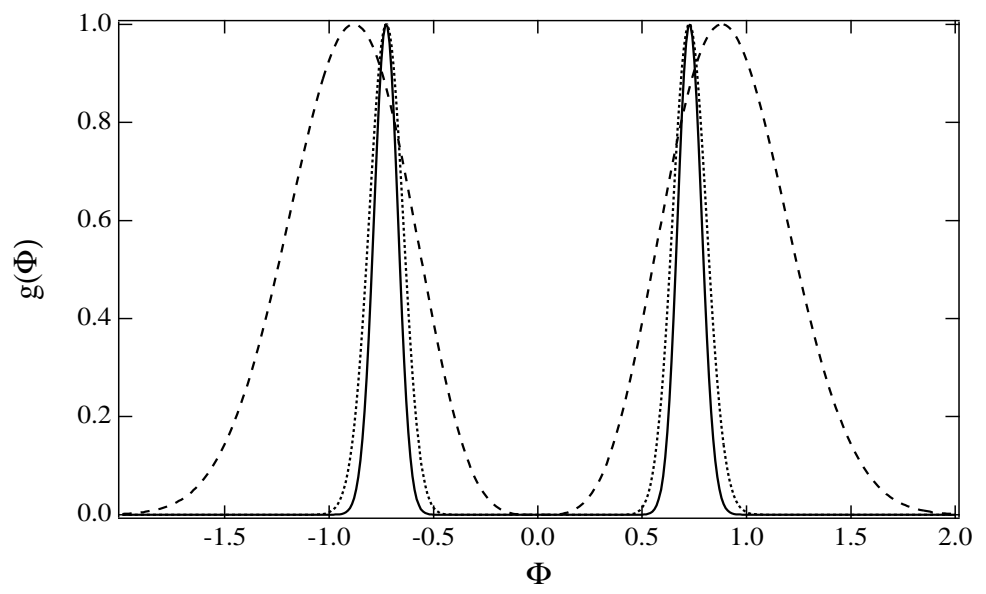

Figure 2: The angular distribution $g(\Phi)$ as a function of angle for three different numbers of measurements of transverse spin, 10 measurements (dashed line), 150 measurements (dottted line), and 300 measurements (solid line). For a single measuring angle this always has two equal peaks, corresponding to the ambiguity of the spin direction with respect to the transverse plane; but making measurements along another direction rapidly removes one of the peaks. The peaks narrow when the number of measurements increases.

The transposition of the question of Leggett and Sols would then be "Can it really be that, by measuring the transverse direction of a few microscopic spins, we can force the macroscopic polarization of $10^{23}$ atoms (or more) to take a definite value?" The analysis of double spin condensates in the present paper shows that, within standard quantum mechanics, one can obtain detailed and exact predictions of the effect of an arbitrary number of measurements in any direction on the macroscopic polarization; the paradox can then be studied in more detail [4. The subject is also related to the discussion of spontaneous symmetry breaking of spin condensates given by Siggia and Rückenstein [24].

\subsection{Transposition of the EPR argument to double condensates}

One usually discusses the standard EPR argument in the form proposed by Bohm, with two spin $1 / 2$ particles entangled in the singlet spin state $\left|S=0, M_{S}=0\right\rangle$, or equivalently in the triplet $\left|S=1, M_{S}=0\right\rangle$. In these situations, the measurement of the spin of the first particle in any direction determines the value of the second spin along the same direction. As soon as the first result is known, the second is also known with certainty when the two directions of measurement are parallel: perfect correlations are predicted by quantum mechanics. This leads EPR to their famous statement : "If, without in any way disturbing a system, we can predict with certainty the value of a physical quantity, then there exists an element of physical reality corresponding to this physical quantity."

With condensates, what emerges from the measurements (still assuming $M \ll N$ ) is a relative phase (Anderson phase) of the condensates, through the process discussed above. Initially, this phase is completely undetermined, and the first spin measurement provides a completely random result. But the 
phase rapidly emerges under the effect of a few measurements, and then remains constant 9 ; it takes a different value for each realization of the experiment, as if the experiment was revealing the pre-existing value of a classical quantity.

Assume now, as in [4], that the double condensate extends over a very large region of space, covering both Alice's laboratory and Bob's very remote laboratory, as shown in Fig. 11. We then have a situation where, without in any way disturbing Bob's system, we can predict from Alice's results the direction of the macroscopic orientation that Bob will observe in his remote laboratory; then there must exist in Bob's laboratory an element of reality associated with this prediction - at this stage, standard quantum mechanics still agrees, provided one uses the postulate of wave packet reduction, which accounts for this element of reality. In addition, since Bob's laboratory is far away and therefore protected from any influence of Alice's operations, the element of reality also necessarily existed before Alice made any measurement - then standard quantum mechanics cannot agree anymore. In fact, it does not only ignore this initial element of reality, but even says that the phase is completely undetermined before the first measurement; the EPR argument then concludes that quantum mechanics is incomplete. We have already emphasized in the introduction that the major difference between this case and the usual two spin case is that, here, the EPR element of reality can be macroscopic; this weakens Bohr's rebuttal of the EPR argument, which hinges on the ambiguity of physical reality for isolated microscopic systems (considered independently of the macroscopic measurement apparatuses), and seems more difficult to transpose to macroscopic systems.

There are also a few other differences. First, with double condensates, a single measurement of the spin of one particle is not sufficient to determine the relative phase; Alice and Bob, have to measure the spin of at least a few particles to obtain a reasonable determination of this phase, with better and better accuracy when the number of measurements increases. This is not a problem, since the total number of available particles may be macroscopic, while a few tens of measurements are already sufficient to obtain an excellent determination; see [25] for a discussion of the strategies that Alice and Bob may use to optimize their knowledge of the phase. We remark in passing that, with condensates, the usual discussion of incompatible measurements, counterfactuality, etc. is not relevant: Alice and Bob can use exactly the same experimental procedures in all realizations of the experiment, and obtain a good knowledge of the phase.

The second difference is that, while for two particles the quantization axis along which both spins polarize is fixed by the direction of first measurement, here the system "chooses for itself" its phase and therefore its quantization axis; the emerging transverse orientation can have any direction with respect to the direction of measurements. Moreover, this direction is only known with an accuracy that is limited by a quantum uncertainty, which decreases when the number of measurements increases (phase/number quantum uncertainty relation). Even if Alice and Bob choose parallel directions for their measurements (or any relative direction), perfect correlations are not predicted in general by quantum mechanics, but only equal probabilities for obtaining result +1 for instance; individual measurements therefore remain stochastic processes so that, strictly speaking, the words "with certainty" used by EPR therefore do not apply with double condensates.

Fortunately, this does not ruin the EPR reasoning: if Alice makes appropriate measurements to determine the phase with good accuracy, and if Bob chooses a direction of measurement that is parallel to the spontaneous transverse magnetization that Alice has observed, the certainty is just replaced by a high probability, 99\% for instance. Alternatively, one can also consider that Alice and Bob use sequences of individual spin measurements to measure the angle of the transverse spin polarization; if these sequences are sufficiently long, there is a high probability that their determinations of the phase will agree within a small error bar. Therefore strong correlations are still obtained in this case, even if no longer at the level of individual measurements. Local realism then ascribes their origin to correlated elements of reality belonging to these remote regions of space, and the essence of the EPR reasoning still applies. We note,

\footnotetext{
${ }^{9}$ As already mentioned, we ignore any evolution of the system between measurements.
} 
nevertheless, that here only one additional element of reality emerges from the EPR reasoning, that associated with the direction that the system has chosen, while in the usual situation with two spins all components of Bob's single spin are predictable from Alice's result (provided she chooses a parallel direction of measurement). But, if one accepts local realism, one missing element of reality is already sufficient to prove that quantum mechanics is incomplete! One can summarize all this discussion by saying that the usual EPR microscopic elements of reality, associated with all components of a single spin, collapse here into one single, macroscopic, element of reality.

\section{Microscopic violations of local realism}

We now continue the EPR reasoning to derive Bell inequalities; we then show that the quantum predictions violate these inequalities; we complete this section with a comparison between violations obtained with GHZ and double Fock states.

\subsection{Bell inequalities within stochastic local realist theories}

The derivation of Bell inequalities from the EPR conclusions involves different reasonings in the usual case (two spins) and for two condensates. We first recall the situation in the usual case.

\subsubsection{Two spins}

With two spins, the derivation of the Bell theorem starts from the existence of well defined functions $A\left(\lambda, \varphi_{a}\right)$ and $B\left(\lambda, \varphi_{b}\right)$ giving the results of the measurements; these functions depend on the fluctuating elements of reality $\lambda$ that each particle carries with it, and of the local orientation $\varphi_{a}$ or $\varphi_{b}$ of the measurement apparatus. Within local realism, their existence is proved by the fact that, for any direction chosen by Alice (or Bob), it is always possible that Bob (or Alice) will choose a parallel direction; one can then predict with certainty the second observed result from another measurement made very far away. The results of spin measurements are therefore deterministic functions of the additional variable $\lambda$ and of the local setting; the original Bell reasoning [5, 6] then leads to the usual Bell inequalities.

The result can be generalized to a stochastic point of view; the inequalities do not require determinism, but can also be proved within stochastic realist theories, provided they are local [20]. We call $P_{+}^{a}\left(\lambda, \varphi_{a}\right)$ the probability that Alice will obtain a result +1 when the relative phase is $\lambda$ and when she has chosen a direction $\varphi_{a}$ for her measurements, $P_{-}^{a}\left(\lambda, \varphi_{a}\right)$ the probability for the opposite result; a similar notation $P_{ \pm}^{b}\left(\lambda, \varphi_{b}\right)$ is used for Bob. For a given realization of the experiment, with a given phase $\lambda$, the expectation of the product of the results is:

$$
P_{+}^{a}\left(\lambda, \varphi_{a}\right) P_{+}^{b}\left(\lambda, \varphi_{b}\right)+P_{-}^{a}\left(\lambda, \varphi_{a}\right) P_{-}^{b}\left(\lambda, \varphi_{b}\right)-P_{+}^{a}\left(\lambda, \varphi_{a}\right) P_{-}^{b}\left(\lambda, \varphi_{b}\right)-P_{-}^{a}\left(\lambda, \varphi_{a}\right) P_{+}^{b}\left(\lambda, \varphi_{b}\right)
$$

The average of the product of the results observed by Alice and Bob in many realizations of the experiment is then:

$$
\langle\bar{A} \bar{B}\rangle=\int_{-\pi}^{+\pi} \frac{d \lambda}{2 \pi}\left[P_{+}^{a}\left(\lambda, \varphi_{a}\right)-P_{-}^{a}\left(\lambda, \varphi_{a}\right)\right]\left[P_{+}^{b}\left(\lambda, \varphi_{b}\right)-P_{-}^{b}\left(\lambda, \varphi_{b}\right)\right]
$$

that is, the average over the possible values of the relative phase $\lambda$ of the product of the two quantities:

$$
\begin{aligned}
& \bar{A}\left(\lambda, \varphi_{a}\right)=P_{+}^{a}\left(\lambda, \varphi_{a}\right)-P_{-}^{a}\left(\lambda, \varphi_{a}\right)=2 P_{+}^{a}\left(\lambda, \varphi_{a}\right)-1 \\
& \bar{B}\left(\lambda, \varphi_{b}\right)=P_{+}^{b}\left(\lambda, \varphi_{b}\right)-P_{-}^{b}\left(\lambda, \varphi_{b}\right)=2 P_{+}^{b}\left(\lambda, \varphi_{b}\right)-1
\end{aligned}
$$

In the second equation of each line, we have taken into account that the sum of probabilities $P_{+}$and $P_{-}$, for given $\lambda$ and angle of measurement, is 1 ; because all probabilities are numbers between 0 and 1 , for any value of $\lambda$ and the angle $\varphi$ both $\bar{A}$ and $\bar{B}$ are numbers between -1 and +1 . 
If now we form the combination of the average that appear in (17), we obtain the average over the phase $\lambda$ of the expression:

$$
\bar{A} \bar{B}+\bar{A} \overline{B^{\prime}} \pm\left(\overline{A^{\prime}} \bar{B}-\overline{A^{\prime}} \overline{B^{\prime}}\right)
$$

where the primes indicate that the angle $\varphi_{a}$ has been replaced by $\varphi_{a}^{\prime}$ (or $\varphi_{b}$ by $\varphi_{b}^{\prime}$ ). The only difference with the deterministic case is that the numbers that appear in (25) are no longer equal to \pm 1 , but have some value between -1 and +1 . But expression (25) is linear with respect to all of these numbers separately. Therefore, if we replace one of the numbers, $\bar{A}$ for instance, by \pm 1 , we change the expression to new values that provide upper and lower bounds of the initial value. Doing the same thing for all four variables in succession therefore provides new lower and upper bounds which, since now all numbers are \pm 1 , are \pm 2 . At the end of the process, we see that (25) is still bound between -2 and +2 ; its average value over the phase $\lambda$ must have the same property, so that the BCHSH equations remain valid.

\subsubsection{Two condensates}

With two condensates, the situation is different: when $N_{+}$and $N_{-}$are more than 1 , equation (14) does not contain situations with full correlations. When individual spin results observed by one of the experimenters cannot be predicted with certainty (for any direction of measurement) from the result already obtained by the other, local realism can no longer be used to derive the existence of functions $A\left(\lambda, \varphi_{a}\right)$ and $B\left(\lambda, \varphi_{b}\right)$. There is no way to force the axis of quantization, as already discussed in $\S 3.1$ the relative phase that emerges from the measurements is independent of the directions of measurements. If, for instance, Alice and Bob choose a common direction that happens to be perpendicular to the transverse direction that has spontaneously appeared, each of them will have $50 \%$ probabilities for the two results, and the EPR element of reality provides them with no information at all. The connection between the EPR reasoning and the Bell theorem can therefore not be directly transposed from the two-spin case.

A way to proceed is to extend the analysis of $\S 2.2$ by a reasoning that we will call the quasi-classical treatment of the relative phase (Anderson phase). We have seen that the local realist EPR argument, applied to sequence of measurements where $M$ remains smaller than the number of particles $N$, leads us to conclude that the sample is fully polarized in some unknown direction. This full polarization has no reason to disappear when more measurements are performed: for instance, if Bob's sample is initially fully polarized, it will keep this full polarization if Alice accumulates more measurements on her side, and even completes the sequence of measurements so that $M$ becomes equal to $N$ : arbitrarily remote experiments can not influence the local physical properties of Bob's sample. So we can consider, within local realism, that both Alice and Bob actually do experiments on fully polarized samples with unknown transverse directions. In this case, for each realization of the experiment, all spins are in the same individual quantum state, and the spin measurements are actually independent processes. We can then write the simple formula:

$$
\mathcal{P}\left(\eta_{1}, \eta_{2}, \ldots \eta_{M}\right)=\int_{-\pi}^{+\pi} \frac{d \lambda}{2 \pi} \prod_{j=1}^{N} P_{\eta_{j}}^{(j)}\left(\lambda, \varphi_{j}\right)
$$

where $P_{\eta_{j}}^{(j)}\left(\lambda, \varphi_{j}\right)$ are the individual spin probabilities, which obey:

$$
P_{+1}^{(j)}\left(\lambda, \varphi_{j}\right)+P_{-1}^{(j)}\left(\lambda, \varphi_{j}\right)=1 \quad ; \quad P_{\eta_{j}}^{(j)}\left(\lambda, \varphi_{j}\right) \geq 0
$$

and where the correlations between the measurements are introduced by the $\lambda$ integral in (26). Of course the simplest idea is to choose for all of them the same probability, for instance that given by quantum mechanics for the measurement on a single spin:

$$
P_{\eta}^{(j)}(\lambda, \varphi)=\frac{1}{2}[1+\eta \cos (\lambda-\varphi)]
$$


but we can also take for this probability an arbitrary function of its variables, provided conditions (27) are fulfilled. In any case, we arrive at a situation where the EPR reasoning leads to probabilities instead of certainties.

Assume now that Alice makes $N_{a}$ measurements and Bob $N_{b}$; formula (26) gives the probability of any series of results they observe. For using the BCHSH formula, both must choose functions $\mathcal{A}$ and $\mathcal{B}$ that depend on their local results, and take values that remain between \pm 1 . There is a large flexibility at this stage: Alice decides to attribute value $\mathcal{A}=+1$ to some some chains of her results $\eta_{1}, \eta_{2}, . . \eta_{N_{a}}$, value $\mathcal{A}=-1$ to all the others; Bob makes a similar choice. Now, to obtain the probability that the product $\mathcal{A B}$ is 1 , we can sum the probabilities of two exclusive events (either $\mathcal{A}=\mathcal{B}=1$, or $\mathcal{A}=\mathcal{B}=-1$ ) and use (26). Let us for instance calculate the probability of the first event which, according to (26) , is the product of two local probabilities, $P_{+}^{a}\left(\lambda ; \varphi_{1}, . . \varphi_{N_{a}}\right)$ and $P_{+}^{b}\left(\lambda ; \varphi_{N_{a}+1}, . . \varphi_{N_{a}+N_{b}}\right)$, defined as:

$$
P_{+}^{a}\left(\lambda ; \varphi_{1}, . . \varphi_{N_{a}}\right)=\sum_{\mathcal{A}=+1} \prod_{j=1}^{N_{a}} P_{\eta_{j}}^{(j)}\left(\lambda, \varphi_{j}\right) \quad ; \quad P_{+}^{b}\left(\lambda ; \varphi_{N_{a}+1}, . . \varphi_{N_{a}+N_{b}}\right)=\sum_{\mathcal{B}=+1} \prod_{j=N_{a}+1}^{N_{a}+N_{b}} P_{\eta_{j}}^{(j)}\left(\lambda, \varphi_{j}\right)
$$

where the two sums are taken over the sequences of $\eta$ 's that realize $\mathcal{A}=1$ for Alice, $\mathcal{B}=1$ for Bob; similar reasonings apply to the other values of $\mathcal{A}$ and $\mathcal{B}$. At this point, we see that we have made the connection with the previous calculation: we can define functions $\bar{A}$ and $\bar{B}$ by replacing in (24) $A$ by $\mathcal{A}$ and $B$ by $\mathcal{B}$, and the rest of the reasoning goes unchanged; the only difference is that each local angle $\varphi_{a}$ and $\varphi_{b}$ is replaced by a series of angles. The essential property remains: each function $\bar{A}$ or $\bar{B}$ still depends only on the local angles chosen by its experimenter, and the BCHSH inequality is still valid.

We conclude that the quasi-classical treatment of the relative phase leads to the BCHSH inequalities; each time we can write the probability of combined measurements in the form (26), where the $P_{\eta_{j}}^{(j)}\left(\varphi_{j}\right)$ are numbers between 0 and 1 , these inequalities hold.

\subsection{Comparison with the quantum predictions}

The quantum predictions of equation (14) are not exactly of the form (26), but they are similar. Are the differences sufficient to introduce violations of local realism? We already know that they are, since we have seen in $\S 2.3$ that the BCHSH inequalities can be violated by the quantum results; here we study in more detail the mechanism of these violations.

First, we have already noted that, if $N$ is large and if $M \ll N$, the peaking effect $[\cos \Lambda]^{N-M}$ selects only the values of $\Lambda$ around zero, so that a good approximation is to take $\Lambda=0$ inside all the brackets contained in the product over $j$; then the $\Lambda$ integral disappears and one exactly recovers (16), so that no violation is possible. For large violations of local realism, the most interesting cases occur when $M$ has its maximal value $N$; so, while in the preceding section we discussed mostly the situation where $M \ll N$, here we are mostly interested in the opposite case.

If $\Lambda$ does not vanish, (14) remains similar to (26), while not identical. The first difference is that (14) contains a double integral, but this is not essential: clearly the results of $\S 4.1$ can easily be generalized to more than one additional variable $\lambda$, for instance two $\lambda$ and $\Lambda$, and to situations where the distribution $\rho(\lambda, \Lambda)$ is not uniform; any positive normalized distribution is possible. If we attempt to bring (14) to a form that is compatible with local realist theories, we must satisfy conditions (27); for this purpose, in the product over $j$, we factorize $[\cos \Lambda]^{M}$ so that each term in the product becomes:

$$
\left[1+\eta_{j} \frac{\cos \left(\lambda-\varphi_{j}\right)}{\cos \Lambda}\right]
$$

Then the sum of probabilities associated with the two $\eta= \pm 1$ results is indeed 1 , as requested. But, at the same time, we see that the "probabilities" introduced in this way may become negative for some values of the variables, which opens the way to violations of the BCHSH inequalities, by a mechanism that we now 
discuss. First, if we define $\mathcal{A}$ and $\mathcal{B}$ as equal to 1 for any value of the variables $\eta$, because the quantum probabilities are normalized to 1 by summing over the results $\eta$ 's, each of the 4 terms contained in $\langle Q\rangle$ is then exactly 1 , so that $\langle Q\rangle=2$. Now suppose that, for some values of the variables, the product of "probabilities" is negative; if we redefine $\mathcal{A}$ and $\mathcal{B}$ in such a way that makes their product negative for these values, this will automatically increase the value of $\langle Q\rangle$ beyond 2 and violate the BCHSH inequalities. In fact, since $\mathcal{A}$ and $\mathcal{B}$ depend only on the $\eta$ 's and not on the other variables $\lambda$ and $\Lambda$, this operation may affect at the same time domains of the variables where the product of "probabilities" is negative, and positive; the net effect is then a balance between positive and negative contributions, and the violations do occur when the contribution of the former outweighs those of the latter. In any case, negative probabilities are a necessary conditions for violations of the inequalities; in $\S$, we discuss in detail several examples of these situations.

\subsection{GHZ states versus double Fock states for violations of local realism}

Mermin [26] has proposed a thought experiment involving many particles and leading to exponential violations of local realism; we now briefly compare his scheme with ours. He uses a maximally entangled spin state (GHZ, or NOON state), which is sometimes considered as the "most quantum state" accessible to an $N$ particle system. The GHZ states are also sometimes called "Schrödinger cat states", since they involve a coherent superposition of states that are macroscopically distinct if $N$ is very large; they are not easy to produce experimentally with many particles - to our knowledge, the world record [27] for the number of particles is $N=5$ - and very sensitive to decoherence [28].

Our double Fock state (1) is the simplest possible state that is compatible with Bose statistics. Conceptually, there is no simpler way to put together identical particles in two different spin states; at first sight, it does not even look entangled but, still, strong violations of the BCHSH inequalities do occur. Reference [29] shows how double Fock states with equal populations can be used in interferometers to measure the relative quantum phase at the Heisenberg limit. Such states also undergo decoherence by coupling to the environment, although more slowly than GHZ states 28]; the "natural basis" for decoherence is given by phase states (corresponding to different macroscopic spin orientations), and its effect on our conclusions are minor, since nothing in the calculations requires coherence between various phase states. With present experimental techniques, there seems to be no enormous difficulty in producing double Bose-Einstein condensates. Nevertheless, to observe the quantum non-local effect we study here, it is essential to obtain the equality $N_{+}=N_{-}$. This means for instance that it is necessary to carefully avoid atom losses in both condensates: values of $N_{+}$and $N_{-}$of the order of 10 seem accessible experimentally, but probably not orders of magnitude more.

A striking feature of Mermin's thought experiment is the exponential violation that is predicted; we obtain nothing similar here, just a violation comparable to Cirelson's limit. Nevertheless it should be realized that, for $N$ particles, the observable that Mermin introduces is the sum of $2^{N-1}$ commuting products of operators. It seems difficult to imagine how to measure this sum without measuring the $2^{N-1}$ commuting components. Seen in this way, the Mermin scheme amounts to accumulating $2^{N-1}$ measurements, and taking a sum of results in a way that accumulates the violation and makes it proportional to the square root of the number of measurements; this procedure can of course be implemented in other schemes, including two-particle experiments, or our scheme, and leads to violations that are even linear in the number of measurements. But the price to pay in all cases is a large increase of the number of measurements.

Finally - and this is probably the most important difference that we have already emphasized — there is an important conceptual difference, since with the GHZ state the EPR "elements of reality" remain microscopic, while with double Fock states they may be macroscopic. 


\section{Types of measurements; numerical results}

Here we consider various types of measurement and the quantitative values of violations that occur with Bose-Einstein condensates. While up to this point we have considered only the usual form of inequality shown in (17), other forms are possible as we see here. Moreover, the quantities $\mathcal{A}, \mathcal{B}$, etc. can take on forms other than a simple product of $\eta$ 's. Values of the inequality violations will be given in this section but angles for the spin measurements will be presented in an Appendix.

\subsection{Products of $\eta$ 's}

We return to (15) in which we computed the average of a product of experimental results for the $\eta_{i}$. Such a product is \pm 1 and so qualifies to be an $\mathcal{A}$ or $\mathcal{B}$. We consider the case where the number of experiments is equal to the total number of particles, $M=N$ and the numbers of up and down spin particles are the same, $N_{+}=N_{-}$. Then we have the simple result:

$$
E\left(\varphi_{1}, \varphi_{2}, . . \varphi_{N}\right)=\frac{\int_{-\pi}^{+\pi} \frac{d \lambda}{2 \pi} \prod_{j=1}^{N} \cos \left(\lambda-\varphi_{j}\right)}{\int_{-\pi}^{+\pi} \frac{d \Lambda}{2 \pi}[\cos \Lambda]^{N}}
$$

We assume Alice makes $P$ measurements, all at the same angle $\varphi_{a}$, and Bob makes $N-P$ at angle $\varphi_{b}$, corresponding to products of results $\mathcal{A}$ and $\mathcal{B} ; \mathcal{A}^{\prime}$ and $\mathcal{B}^{\prime}$ correspond to two other values of the angles $\varphi_{a}^{\prime}$ and $\varphi_{b}^{\prime}$. Equation (31) then reduces to:

$$
E\left(\varphi_{a}, \varphi_{b}\right)=\frac{\int_{-\pi}^{+\pi} \frac{d \lambda}{2 \pi} \cos ^{P}\left(\lambda-\varphi_{a}\right) \cos ^{N-P}\left(\lambda-\varphi_{b}\right)}{\int_{-\pi}^{+\pi} \frac{d \Lambda}{2 \pi}[\cos \Lambda]^{N}}
$$

Then the quantum average for the Bell test of the inequality (17) is:

$$
\langle Q\rangle=E\left(\varphi_{a}, \varphi_{b}\right)+E\left(\varphi_{a}^{\prime}, \varphi_{b}\right)+E\left(\varphi_{a}, \varphi_{b}^{\prime}\right)-E\left(\varphi_{a}^{\prime}, \varphi_{b}^{\prime}\right)
$$

In the numerator of (32) we change variables to $\lambda^{\prime}=\lambda-\varphi_{b}$; if we define:

$$
\chi=\varphi_{a}-\varphi_{b}
$$

we obtain:

$$
\begin{aligned}
E\left(\varphi_{a}, \varphi_{b}\right) & \sim \int_{-\pi}^{+\pi} \frac{d \lambda^{\prime}}{2 \pi} \cos ^{P}\left(\lambda^{\prime}-\chi\right) \cos ^{N-P}\left(\lambda^{\prime}\right)=\int_{-\pi}^{+\pi} \frac{d \lambda^{\prime}}{2 \pi} \cos ^{N-P}\left(\lambda^{\prime}\right)[\cos \lambda \cos \chi+\sin \lambda \sin \chi]^{P} \\
& =\sum_{k=0}^{q}\left(\begin{array}{l}
P \\
k
\end{array}\right) \sin ^{k} \chi \cos ^{P-k} \chi \int_{-\pi}^{+\pi} \frac{d \lambda^{\prime}}{2 \pi} \cos ^{N-k} \lambda^{\prime} \sin ^{k} \lambda^{\prime}
\end{aligned}
$$

The integral is known and we find:

$$
E(\chi)=\frac{(N / 2) !}{N !} \sum_{k=0,1}^{\{P / 2\}} \frac{P !(N-2 k) !}{k !(P-2 k) !\left(\frac{N}{2}-k\right) !} \sin ^{2 k} \chi \cos ^{P-2 k} \chi
$$

where $\{P / 2\}$ is the integral part of $P / 2$. This result is efficiently evaluated in numerical maximizations of $\langle Q\rangle$, so that rather large $N$ values can be treated. We always find a fan arrangement with $\varphi_{a}-\varphi_{b}=$ $\varphi_{b}-\varphi_{a^{\prime}}=\varphi_{b^{\prime}}-\varphi_{a}=\chi$ and $\varphi_{b^{\prime}}-\varphi_{a^{\prime}}=3 \chi$, although the value of $\chi$ at maximum decreases with increasing $N$ - see the Appendix. 
For arbitrary $N$ and $P=1$, we again see that $E(\chi)=\cos \chi$ as noted above in (19), yielding $\langle Q\rangle_{\max }=$ $2 \sqrt{2}$. For $P=2$, the result is:

$$
E(\chi)=\frac{1}{2}\left[1+\frac{1}{N-1}+\left(1-\frac{1}{N-1}\right) \cos 2 \chi\right]
$$

so that $\langle Q\rangle_{\max }$ will depend on $N$. For $N=4$ the value is 2.28 , but for very large $N$ we obtain $\langle Q\rangle_{\max }=$ 2.414; surprisingly it increases with $N$. In Fig. 3 we plot $\langle Q\rangle_{\max }$ versus $N$ for various $P$ values; even in the case $P=N / 2$, we still get violations for all $N$.

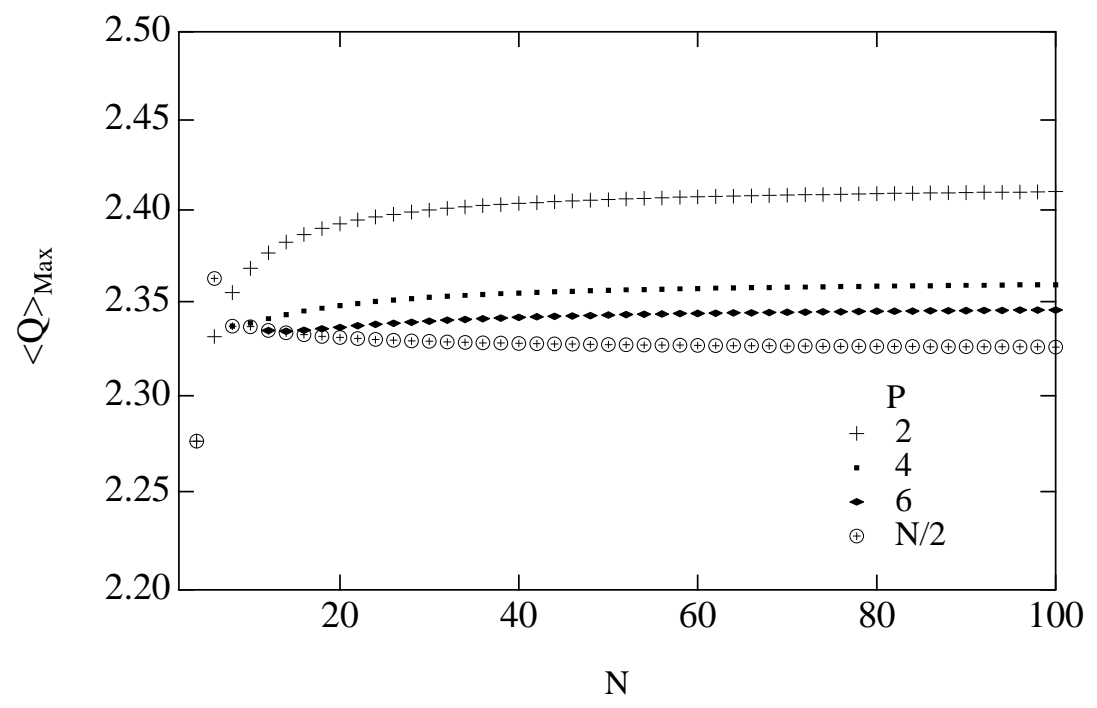

Figure 3: The maximum of the quantum average $\langle Q\rangle$ for Alice doing $P$ experiments and Bob $N-P$, as a function of the total number of particles $N$. Local realist theories predict an upper limit of 2; large violations of this limit are obtained, even with macroscopic systems $(N \rightarrow \infty)$. Not shown is the case $P=1$ for which the Cirel'son limit of $2 \sqrt{2}$ is obtained for all $N$.

In the case in which both $P$ and $N-P$ are very large, a simple approximation for $E$ is available. By expanding the logarithm of $\cos ^{L} \chi$ to second order in $\chi$ we obtain the approximate form:

$$
\cos ^{L} y \cong e^{-\frac{L}{2} y^{2}}
$$

from which we find:

$$
E\left(\varphi_{a}, \varphi_{b}\right) \cong \frac{\int_{-\infty}^{+\infty} d \lambda e^{-\frac{P}{2}\left(\lambda-\varphi_{a}\right)^{2}} e^{-\frac{N-P}{2}\left(\lambda-\varphi_{b}\right)^{2}}}{\int_{-\infty}^{+\infty} d \lambda e^{-\frac{N}{2}(\lambda)^{2}}}=e^{-\frac{P(N-P)}{2 N} \chi^{2}}
$$

In the case $P=N / 2$ we maximize a sum of Gaussians, and again find a fan arrangement, with a maximum of $8 /\left(3 \times 3^{1 / 8}\right)=2.32$ at $\chi=\sqrt{\ln 3 / N}$. So the fan opening decreases as $1 / \sqrt{N}$; this approximate result is valid for $N$ as small as 12. This is similar to the results obtained by Drummond 30, who nevertheless starts from a many particle quantum state that is very different from ours (he considers $\mathrm{N}$ identical pairs of particles, each pair in a state that involves 4 single-particle states) as well as a different measurement scheme (number of bosons in one spin state, at two different locations). 
We have also relaxed the constraint that all the angles within a set of measurements providing $\mathcal{A}$, or those providing $\mathcal{B}$, etc. be identical. Thus we might introduce $\varphi_{a 1}, \varphi_{a 2}$, etc. and $\varphi_{b 1}, \varphi_{b 2}$, etc. We have numerically examined such generalizations for $N$ up to 10, and found that the maximization in this larger space collapses to the one we discuss above, $\varphi_{a 1}=\varphi_{a 2}=\cdots=\varphi_{a}$, with a single angle for all $\mathcal{A}$ measurements, and a single one for all $\mathcal{B}$ measurements, etc.

Experiments with $M<N$ do not result in violations of the Bell inequalities. To see this write equation (15) in the form:

$$
E\left(\varphi_{1}, \varphi_{2}, . . \varphi_{M}\right)=\frac{\int_{-\pi}^{+\pi} \frac{d \lambda}{2 \pi} \prod_{j=1}^{M} \cos \left(\lambda-\varphi_{j}\right)}{\int_{-\pi}^{+\pi} \frac{d \lambda}{2 \pi} \cos ^{M} \lambda} G\left(M, N_{+}, N_{-}\right)
$$

where, if $M$ is even:

$$
\begin{array}{r}
G\left(M, N_{+}, N_{-}\right)=\frac{\int_{-\pi}^{+\pi} \frac{d \Lambda}{2 \pi} \cos \left[\left(N_{+}-N_{-}\right) \Lambda\right] \cos ^{N-M} \Lambda \int_{-\pi}^{+\pi} \frac{d \lambda}{2 \pi} \cos ^{M} \lambda}{\int_{-\pi}^{+\pi} \frac{d \Lambda}{2 \pi} \cos \left[\left(N_{+}-N_{-}\right) \Lambda\right] \cos ^{N} \Lambda} \\
=\frac{(N-M) ! M ! N_{+} ! N_{-} !}{\left(N_{+}-\frac{M}{2}\right) !\left(N_{-}-\frac{M}{2}\right) !\left(\frac{M}{2} !\right)^{2} N !}
\end{array}
$$

and $G=0$ if $M$ is odd. The first factor in equation (40) is the expectation value for $M$ experiments with $M$ particles and can lead to a violation of the Bell inequality. But the correction term $G$ can be shown, for fixed $M$, to be largest for $N_{+}=N_{-}=N / 2$; then an analysis of $G(M, N / 2, N / 2)$ shows that this quantity is always less than or equal to $2 / 3$ unless $M=N$. Since $(2 / 3) 2 \sqrt{2}<2$, we have the remarkable result that one must measure every particle's spin in order to see a violation of the Bell inequality. Even missing the measurement of one or two particles ruins the observations of the quantum effect.

\subsection{Other definitions of $\mathcal{A}$ and $\mathcal{B}$}

In the above analysis, we have used only a product of all the $\eta^{\prime} s$ as the $\mathcal{A}$ or $\mathcal{B}$ quantity. Other possibilities are available. For example, if Alice and Bob each make $N / 2$ measurements, we might take $\mathcal{A}$ and $\mathcal{B}$ in the form:

$$
\frac{\eta_{1}+\eta_{2}+\cdots \eta_{N / 2}}{\left|\eta_{1}+\eta_{2}+\cdots \eta_{N / 2}\right|}
$$

which, if both Alice and Bob choose one single angle of measurement, would be a macroscopic polarization of the spins measured by each; more precisely, the numerator of this expression is the macroscopic polarization (in dimensionless units), and the denominator ensures a "binning" operation that retains only the sign \pm 1 . Averaging the product of the two polarizations for $\langle\mathcal{A B}\rangle$, we found that this procedure does not lead to a Bell violation for any set of angles, except of course for $N=2$ where the violation is $2 \sqrt{2}$. For $N=4$, we find $\langle Q\rangle_{\max }=1.88$; for $N=8$, we find 1.78 ; for $N=10$, we find $1.970 ;$ for $N=14$, we find 1.966; the value seems to converge asymptotically to 2 , that is, the upper limit of local realism.

On the other hand, if Alice makes $N-1$ measurements and Bob just one, the average of the product of her polarization and his single value:

$$
\frac{\eta_{1}+\eta_{2}+\cdots \eta_{N-1}}{\left|\eta_{1}+\eta_{2}+\cdots \eta_{N-1}\right|} \eta_{N}
$$

does lead to violations for one value of $N$ only. The values for $N=4,6,8,19$ are respectively, $\langle Q\rangle_{\max }=$ $1.41,2.121,1.59,1.99$. There is a violation for $N=6$ with higher values again possibly tending to 2 . This violation is a remarkable result since, here, Alice makes a measurement that is almost mesoscopic; the quantum character of Bob's measurement is nevertheless sufficient to maintain a significant violation of local realism. Nevertheless, if the number of measurements made by Alice increases beyond $N=6$, the violations disappear. Other cases where Bob makes two or more measurements, and Alice the complement to $N$, do not lead to violations. 
We have also tried considering averages of sums of two measurements in the form of products of $\left(\eta_{1}+\eta_{2}\right) / 2$. Such a quantity has possible values $1,0,-1$ but the Bell inequality still holds. We found no cases where the quantum average of such pair averages lead to a violation.

\subsection{Other inequalities}

Other inequalities besides that of (17) are possible. For example, consider the inequality:

$$
-2 \leq \frac{1}{2}\left(\mathcal{A B}+\mathcal{A}^{\prime} \mathcal{B}+\mathcal{A B}^{\prime}-\mathcal{A}^{\prime} \mathcal{B}^{\prime}\right)\left(\mathcal{C D}+\mathcal{C}^{\prime} \mathcal{D}+C D^{\prime}-\mathcal{C}^{\prime} \mathcal{D}^{\prime}\right) \leq 2
$$

where each of the letters represents an $\eta$ or a product of any number of $\eta$ 's. We assume that the angles of measurement corresponding to the $\eta$ 's in each letter are all the same (but releasing this constraint in the corresponding quantum average does not increase the violation found.). For $N=4$ each letter represents just one $\eta$, while for $N=8$ each letter corresponds to the product of two $\eta$ 's. For $N=6$, $\mathcal{A}$ would represents one $\eta$ and $\mathcal{B}$ two, etc. We find the violations shown in Table I when maximizing the corresponding quantum averages (the $N=\infty$ result in Table I comes from making a Gaussian approximation, as in equation (38), for the powers of cosines in the integrals).

Table I. Results for the $\langle Q\rangle_{\max }$ corresponding to the inequality of (44)

$\begin{array}{ll}N & \langle Q\rangle \\ 4 & 2.66 \\ 6 & 2.33 \\ 8 & 2.18 \\ 12 & 2.17 \\ \infty & 2.15\end{array}$

An extension of the idea in (44) is the inequality:

$$
-2 \leq \frac{1}{4}\left(\mathcal{A B}+\mathcal{A}^{\prime} \mathcal{B}+\mathcal{A B}^{\prime}-\mathcal{A}^{\prime} \mathcal{B}^{\prime}\right)\left(\mathcal{C D}+\mathcal{C}^{\prime} \mathcal{D}+\mathcal{C} \mathcal{D}^{\prime}-\mathcal{C}^{\prime} \mathcal{D}^{\prime}\right)\left(\mathcal{E F}+\mathcal{E}^{\prime} F+\mathcal{E} \mathcal{F}^{\prime}-\mathcal{E}^{\prime} \mathcal{F}^{\prime}\right) \leq 2
$$

The quantum counterpart of this yields 2.66 for $N=6$; we therefore obtain a large quantum violation of this particular inequality, which therefore provides an interesting generalization of the BCHSH inequality. We have been able to treat larger $N$ values in this case only by use of the Gaussian approximation discussed earlier. The violation continues for larger $N$ with a limit of $\langle Q\rangle_{\max }=2.09$.

\section{Sample bias (efficiency) loophole}

Our quantum calculations are consistent only if the "measurement boxes" are spatially disconnected (to ensure commutation of the quantum field operators) and if their volume $\Delta$ is sufficiently small to limit the number of particles in each of them to 0 or 1 ; otherwise, the expressions of the projectors we use are not valid 10 . This means that the average number of particles in each box is much less than one, so that most measurements detect no spin at all. But, if one counts 0 for all these non-detection events, clearly the quantum average of the product of results becomes very close to zero, and no violation of the Bell inequalities remains possible!

This is not an unusual situation in Bell-type experiments. When detecting pairs of photons for instance, most photons are lost because of the finite solid angle that is captured by the detectors and of their limited

\footnotetext{
${ }^{10}$ The expression we use is actually the total amount of spin orientation within the volume (in $\hbar / 2$ units). If two particles are found inside the same volume $\Delta$, then the two possible values of this orientation are \pm 2 (instead of \pm 1 for one particle), leading to eigenvalues 2 or 0 of the (space integrated) operator (5). This is in contradiction with the eigenvalues 1 or 0 of a projector; moreover, values exceeding 1 are in contradiction with the assumptions leading to the Bell inequalities.
} 
quantum efficiency. This is known as the "sample bias loophole", "efficiency loophole", "pair selection loophole", etc. To avoid the problem, what is done in practice by experimentalists is to redefine the sample of events in the calculation of the averages: instead of the sample of all emitted pairs, they consider the sample of pairs for which particles in coincidence are indeed detected. This restores the possibility of a violation of the inequalities, but at the same time destroys the validity of the BCHSH inequalities themselves, since local realism stricto sensu is then no longer sufficient to derive them. The reason is that there is no way to ensure that the sample remains independent of the settings of the apparatuses, while this assumption is crucial for the proof of the Bell theorem: if the "settings" introduce a bias in the sample, the distribution of variables $\lambda$ may depend on them, and the proof of the theorem becomes impossible 11. One then has to introduce extra assumptions, for instance that the measured probability is the product of a probability of detection (independent of the settings) by a spin (and setting) dependent probability that is relevant to the Bell inequality violation. This experimental loophole has been pointed out many times, and some authors (a minority) have even refuted all locality experiments for this reason; there is a large amount of literature on the subject.

Fortunately, at least for thought experiments, the loophole can be closed; it is therefore not a fundamental obstacle, but only contingent on our present technologies. John Bell had a elegant way to solve the problem 8, with the introduction of either "veto detectors" or "spin independent preliminary detectors"; the purpose of these detectors was to properly define a sample of systems that is independent of the settings and ensures that a spin signal is always obtained at each detector. Similarly, Clauser and Shimony 20] introduce "event ready detectors" that have the same function. In our case with BoseEinstein condensates, we need something similar. The simplest idea is to assume that, before any spin measurement, spin-independent detectors are used to ensure that one particle (and one exactly) is found in each measurement box. It may be necessary to repeat the preparation procedure many times before this result is obtained, since in most cases no particle is found in at least one of the measurement boxes, but in theory this is not a problem: it is sufficient to ignore these cases, and to repeat the preparation stage as many times as needed until the desired result is observed. Only after this sample preparation stage has been successful will the spin measurements be performed. Alternatively, one can decide to replace the initial quantum state, the double Fock state, by the new state obtained after wave packet reduction is applied after a positive preparation stage. Here we study the explicit form of this new initial quantum state.

\subsection{Calculating a new quantum state}

The initial double Fock state is given by (1); we now assume that $M$ "measurement boxes" are defined in the volume occupied by the orbital wave function, and decompose this wave function as:

$$
u(\mathbf{r})=\sum_{m=1}^{M+1} x_{m} \bar{u}_{m}(\mathbf{r})
$$

where for $m \leq M$ the function $\bar{u}_{m}(\mathbf{r})$ is the normalized "projection" 12 of the wave function $u(\mathbf{r})$ into the measurement box number $m ; \bar{u}_{M+1}(\mathbf{r})$ is defined as the complementary projection of $u(\mathbf{r})$ outside all the measurement boxes. The $x_{m}$ are the components of the linear decomposition of $u(\mathbf{r})$ onto the contents of the various boxes, with:

$$
\sum_{m=1}^{M+1}\left|x_{m}\right|^{2}=1
$$

\footnotetext{
${ }^{11}$ It is even possible to show that a selection of detected pairs that is dependent on $\phi_{a}$ and $\phi_{b}$ makes it possible to reproduce any correlation of results within local realist models. In other words, local realism does not introduce inequalities anymore.

${ }^{12}$ This projection is equal to $u(\mathbf{r})$ within the box, zero outside, and then normalized to 1 .
} 
For $m \leq M$, the smaller the measurement boxes, the larger the values of the normalized $\bar{u}_{m}(\mathbf{r})$ 's inside their boxes, and the smaller the coefficients $x_{m}$; for $m=M+1, x_{M+1}$ then remains close to 1 . The creation operators for the states $\mid u,+>$ and $\mid u,->$ can be expressed as functions of the creation operators $a_{\bar{u}_{m},+}^{\dagger}$ and $a_{\bar{u}_{m},-}^{\dagger}$ for the the states $\mid \bar{u}_{m},+>$ and $\mid \bar{u}_{m},->$ :

$$
a_{u,+}^{\dagger}=\sum_{m=1}^{M+1} x_{m} a_{\bar{u}_{m},+}^{\dagger} \quad ; \quad a_{u,-}^{\dagger}=\sum_{m=1}^{M+1} x_{m} a_{\bar{u}_{m},-}^{\dagger}
$$

so that:

$$
|\Phi\rangle=\left[\sum_{m=1}^{M+1} x_{m} a_{\bar{u}_{m},+}^{\dagger}\right]^{N_{+}}\left[\begin{array}{ll}
\sum_{m^{\prime}=1}^{M+1} x_{m^{\prime}} & a_{\bar{u}_{m^{\prime}},-}^{\dagger}
\end{array}\right]^{N_{-}}|\mathrm{vac}\rangle .
$$

All operators in this expression commute, so that usual algebraic expansions of the powers of sums can be used without special care.

We see in (49) that the double condensate state vector contains components on states where the number of particles in each box varies substantially. If, for instance, one selects inside both sums only the terms $m=M+1$, all particles go to the complementary box, while all measurement boxes remain empty; no particle can be detected at all by any of the apparatuses. If, on the other hand, one selects only terms corresponding to a given measurement box, all particles accumulate into this particular box, while all the others remain empty. Of course, one can also spread the particles among all measurement boxes, and what we wish is to consider situations where they are equally filled.

We then decide to introduce a new initial state by retaining from (49) only the components where each measurement box contains one particle exactly. In other words, we project $\mid \Phi>$ onto the subspace where each measurement box contains one particle, and obtain a new state vector $\mid \bar{\Phi}>$. Mathematically, this vector could be written with the introduction of exponentials and integrations into (49), but for simplicity we do not write this expression; what is important here is not so much the mathematical form of the new initial state $\mid \bar{\Phi}>$, but the fact that it exists and can be built, without changing the relative probabilities calculated with $\mid \Phi>$ for detecting single spins in each measurement box. This is true by construction: one can easily see that that all components of $\mid \Phi>$ that have been eliminated from $\mid \bar{\Phi}>$ play no role whatsoever in the calculation of the probability for single particle detection; they just eliminate no-detection and multiple detection events. The only difference is the normalization of the ket, which is changed by the removal of all these useless components; the remaining components must be increased to restore normalization. Physically, since we are now sure that one particle, and one particle only, will be detected in each box, we know that the probabilities for all possible results $\eta_{m}= \pm 1$ add to 1 , which is exactly what we wish for a violation of the inequalities.

\subsection{Discussion}

Expression (49) is nothing but the product of $N_{+}$sums associated with internal state + by $N_{-}$sums associated with the other with internal state - . One can see the physical system as the juxtaposition of two entangled subsystems, one corresponding to the content of the measurement boxes (system I), the other to the content of the complementary box (system II). But, if we limit the discussion to the case where $M$ has its maximal value $N_{+}+N_{-}$(we have seen in $\S 5$ that this corresponds to maximal violations of the BCHSH inequalities), then system II becomes empty; its quantum state is independent of that of the $M$ measurement boxes and factorizes out. System I is then in a pure state, with components that depend on how the two internal states are distributed among the measurement boxes; the number of possibilities is:

$$
\frac{N !}{\left(N-N_{+}\right) ! N_{+} !}=\frac{N !}{\left(N-N_{-}\right) ! N_{-} !}
$$


In state $\mid \bar{\Phi}>$, by construction, both Alice and Bob perform local experiments on a fixed total number of particles, but with a fluctuating number of spins + and spins - This remark allows us to come back to the situation shown in the lower part of figure 1. When the wave function of each condensate is coherently split into two disconnected parts, each spin system remains a single condensate with an orbital function that is the coherent sum of two distant components, and the number of particles in each of these components has large fluctuations. Therefore, neither Alice nor Bob knows the number of spin up, or spin down, that she/he receives; the corresponding fluctuations are essential for the interesting quantum non-local effects to occur. The total number of particles contained in Alice's sample also has large fluctuations in state $|\Phi\rangle$, but since this state can be replaced by $\mid \bar{\Phi}>$ without affecting our results, we see that these fluctuations are not essential; in state $\mid \bar{\Phi}>$, the fluctuations between the numbers of spins up and spins down in each region of space are correlated in a way that cancels the fluctuations of the total number of particles in this region, without destroying the non-local effects.

Rather than writing the resulting state for system I in more detail with identical particles, it is simpler to consider now distinguishable particles.

\section{Violations of local realism with distinguishable particles}

We now show that our results for violations of the Bell inequalities are not just limited to Bose-Einstein condensates but also apply to distinguishable particles. When the number of measurements $M$ has its maximal value $N=N_{+}+N_{-}$, state $\mid \bar{\Phi}>$ corresponds to all particles localized in different boxes, with no spatial overlap, so that they do behave as distinguishable particles; if we wish, we can number them in the state vector by assigning them the number of the box they occupy; this operation does not affect the physical predictions.

\subsection{Quantum state}

Using the numbering of the boxes in which the particles are contained, the state $|\bar{\Phi}\rangle$ can then be written as a product:

$$
|\bar{\Phi}\rangle=\left|\Psi_{\text {orb. }}(1,2, . . N)\right\rangle\left|\Psi_{\text {spin }}(1,2, . . N)\right\rangle
$$

with:

$$
\left.\left.\left|\Psi_{\text {orb. }}(1,2, . . N)\right\rangle=\mid u_{1}(1) u_{2}(2) . . u_{N}(N)\right)\right\rangle
$$

(particle 1 is inside measurement box 1, particle 2 inside measurement box 2, etc.) and:

$$
\left|\Psi_{\text {spin }}(1,2, . . N)\right\rangle=\left|1:+; \ldots N_{+}:+; N_{+}+1:-\ldots . . N:-\right\rangle+\text { permutations }
$$

In this spin state, the $N_{+}$spin up orientations and the $N_{-}$spin down orientations are distributed in all possible ways among the numbered particles.

If we wish, we can completely ignore the factorized orbital state and consider only the spins. For instance, with $N_{+}=2$ and $N_{-}=1$, the spin state reads as:

$$
\left|\Psi_{\text {spin }}(1,2,3)\right\rangle=\frac{1}{\sqrt{3}}[|1:+; 2:+; 3:-\rangle+|1:+; 2:-; 3:+\rangle+|1:-; 2:+; 3:+\rangle]
$$

or, in a more condensed notation:

$$
\left|\Psi_{\text {spin }}(1,2,3)\right\rangle=\frac{1}{\sqrt{3}}[|+,+,-\rangle+|+,-,+\rangle+|-,+,+\rangle]
$$

On the other hand, if $N_{+}=N_{-}=2$, this spin state is, with the same notation:

$$
\begin{array}{r}
\left|\Psi_{\text {spin }}(1,2,3,4)\right\rangle=\frac{1}{\sqrt{6}}[|+,+,-,-\rangle+|+,-,+,-\rangle+|-,+,-,+\rangle+ \\
+|-,-,+,+\rangle+|+,-,-,+\rangle+|-,+,+,-\rangle]
\end{array}
$$


etc. Such spin functions having equal amplitudes for all permutations belong to the category of W-states [31, 32, 33. They can also be seen as the various $M$ components of the "ferromagnetic states" [34] | $J, M>$ of total angular momentum 13 with the maximal possible value $J=N / 2$.

Our study shows that W-states are directly related to double Fock states and suggests a method to create them: start from a spin condensate and then perform a preliminary localization of the particles.

\subsection{Recovering the results obtained with identical particles}

We now check that a calculation with numbered spins allows us to recover our preceding results, without having to worry about orbital variables and symmetrization. For spin numbered $j$, the projector over the eigenstate corresponding to a result $\eta_{j}= \pm 1$ for a measurement along azimuthal direction $\varphi_{j}$ is:

$$
P_{\eta_{i}}^{s p i n}\left(\varphi_{j}\right)=\frac{1}{2}\left[1_{j}+\frac{\eta_{1}}{2}\left(e^{-i \varphi_{j}} \sigma_{j}^{+}+e^{i \varphi_{j}} \sigma_{j}^{-}\right)\right]
$$

with the usual notation $\sigma_{j}^{ \pm}$for the angular momentum operator of spin $j$ and $1_{j}$ for the identity operator, which is the sum of the projectors over the two spin states $|+\rangle$ and $|-\rangle$ :

$$
1_{j}=|+\rangle\left\langle+\left.\right|_{j}+\mid-\right\rangle\left\langle-\left.\right|_{j}\right.
$$

The probability of a sequence of a results $\eta_{1}, \eta_{2}, . . \eta_{N}$ for measurements along polar directions $\varphi_{1}, \varphi_{2}$, $\ldots \varphi_{N}$ is then proportional to:

$$
\left\langle\Psi _ { \text { spin } } ( 1 , N ) \left|\prod _ { j = 1 } ^ { N } \frac { 1 } { 2 } \left[|+\rangle\left\langle+\left.\right|_{j}+\mid-\right\rangle\left\langle-\left.\right|_{j}+\frac{\eta_{1}}{2}\left(e^{-i \varphi_{j}} \sigma_{j}^{+}+e^{i \varphi_{j}} \sigma_{j}^{-}\right)\right]\left|\Psi_{\text {spin }}(1, N)\right\rangle\right.\right.\right.
$$

In this expression, each factor of the product of $N$ brackets contains four terms, each with an operator that gives non zero only if it acts on one of the two states $|+\rangle$ and $|-\rangle$; if a given choice among these four terms is made inside each bracket, a non-zero result is obtained for only one state for the $N$ spins. For instance, if $|-\rangle\left\langle-\left.\right|_{1}, e^{-i \varphi_{2}} \sigma_{2}^{+}, e^{i \varphi_{3}} \sigma_{3}^{-}\right.$, and $\left.\mid+\right\rangle\left\langle+\left.\right|_{4}\right.$, etc. are selected, the spin state has to be $|-,-,+,+, \ldots\rangle$. To obtain a non-zero result, a first condition is then that this state must have a non-zero component in the ket $\left|\Psi_{\text {spin }}\right\rangle$. To ensure that this condition is satisfied, we can multiply all $|+\rangle\langle+|$ 's and $\sigma^{-}$'s by $e^{i \Lambda}$, all $|-\rangle\langle-|$ 's and $\sigma^{+}$'s by $e^{-i \Lambda}$, and calculate the integral of the function $F(\Lambda)$ obtained in this way by:

$$
\int_{-\pi}^{+\pi} \frac{d \Lambda}{2 \pi} e^{i\left(N_{+}-N_{-}\right) \Lambda} F(\Lambda)
$$

But a second condition is that the product with the bra $\left\langle\Psi_{\text {spin }}(1, . ., N)\right|$ must not vanish either, which is the case if the effect of the successive $\sigma^{+}$and $\sigma^{-}$operators flips the same number of spins in each direction. To ensure this, we multiply all $\sigma^{+}$'s by $e^{i \lambda}$, all the $\sigma^{-}$'s by $e^{-i \lambda}$, and introduce a second integral over $\lambda$ :

$$
\int_{-\pi}^{+\pi} \frac{d \lambda}{2 \pi}
$$

If these two conditions are satisfied, one always obtains a non-zero result, actually always the same number since all non-zero components of the state vector are equal. Finally, the probability is proportional te 14 :

$$
\sim \int_{-\pi}^{+\pi} \frac{d \Lambda}{2 \pi} e^{i\left(N_{+}-N_{-}\right) \lambda} \int_{-\pi}^{+\pi} \frac{d \lambda}{2 \pi} \prod_{j=1}^{N} \frac{1}{2}\left[e^{i \Lambda}+e^{-i \Lambda}+\eta_{1}\left(e^{i\left(\lambda-\Lambda-\varphi_{j}\right)}+\text { c.c. }\right)\right]
$$

\footnotetext{
${ }^{13}$ This property is only true for $1 / 2$ spins; the proof can easily be obtained by recurrence, by applying several times operator $J^{-}$onto $\mid J, J>$.

${ }^{14}$ Factors 2 disappear because, for instance, $\sigma^{-}|+>=2|->$.
} 
or:

$$
\sim \int_{-\pi}^{+\pi} \frac{d \Lambda}{2 \pi} \cos \left(N_{+}-N_{-}\right) \Lambda \int_{-\pi}^{+\pi} \frac{d \lambda}{2 \pi} \prod_{j=1}^{N}\left[\cos \Lambda+\eta_{1} \cos \left(\lambda-\Lambda-\varphi_{j}\right)\right]
$$

which is identical to (12) with a trivial change of integration variable. We have therefore recovered the results obtained previously with identical particles, but with numbered spins, as usual in calculations of Bell inequalities violations. Our results are therefore not limited to Bose-Einstein condensates; we have a systematic way to go from identical to distinguishable particles. When the number of measurements $M$ is less than the number of particles $N$, a summation over the results $\eta_{j}$ of the $N-M$ unperformed measurements provides the probability, as in $\S 2.1$

\subsection{Triplet state}

In the case $N=M=2$, the initial state is the triplet state:

$$
\left|\Psi_{\text {spin }}(1,2)>=\frac{1}{\sqrt{2}}[|+,-\rangle+|-,+\rangle]\right.
$$

and we obtain:

$$
\sim \int_{-\pi}^{+\pi} \frac{d \Lambda}{2 \pi} \int_{-\pi}^{+\pi} \frac{d \lambda^{\prime}}{2 \pi}\left[\cos \Lambda+\eta_{1} \cos \left(\lambda^{\prime}-\varphi_{1}\right)\right]\left[\cos \Lambda+\eta_{2} \cos \left(\lambda^{\prime}-\varphi_{2}\right)\right]
$$

or:

$$
\sim 1+\eta_{1} \eta_{2} \cos \left(\varphi_{1}-\varphi_{2}\right)
$$

or, after a normalization to 1 of the sum of the four different probabilities:

$$
P_{\eta_{1}, \eta_{2}}=\frac{1}{4}\left[1+\eta_{1} \eta_{2} \cos \left(\varphi_{1}-\varphi_{2}\right)\right]
$$

This is the usual result, which can also be written as:

$$
P_{\eta_{1}, \eta_{2}}=\frac{1}{4} \int_{-\pi}^{+\pi} \frac{d \lambda^{\prime}}{2 \pi}\left[1+\eta_{1} \sqrt{2} \cos \left(\varphi_{1}-\lambda^{\prime}\right)\right]\left[1+\sqrt{2} \eta_{2} \cos \left(\varphi_{2}-\lambda^{\prime}\right)\right]
$$

In this expression, we see that the brackets inside the integral can indeed become negative, allowing a possible violation of the Bell inequalities.

\section{Conclusion}

Transverse spins measurements on double Fock states provide an interesting case where one can calculate exactly the predictions of quantum mechanics in all experimental situations, even if the measurements depend on many parameters. Another interesting flexibility arises from the choice of the two functions $\mathcal{A}$ and $\mathcal{B}$, which can be defined in different ways; depending on this definition, the physical quantity on which locality is tested is microscopic, macroscopic, or intermediate; one can in this way study in detail the emergence of local classical properties of physical systems from microscopic non-locality, within the formalism of quantum mechanics, as a function of all the parameters of the experiment.

One often rightly emphasizes that quantum entanglement is the essential ingredient of violations of local realism by quantum mechanics; the best known example of strongly entangled quantum states are the GHZ/NOON states, which indeed lead to strong violations. It is nevertheless interesting to note that this maximal entanglement is not a necessary condition; in fact, the minimum correlations that are compatible with Bose-Einstein statistics are already sufficient to lead to strong quantum non-local effects. 
Conversely, this does not mean that statistical effects are a necessary condition for violations either; in fact, we have shown that the same effects can be obtained with distinguishable spins in states belonging to the family of $\mathrm{W}$ states. A common property of all our results is that, in all cases, it is essential to perform the measurements on all particles; if a single one is missed, the violations disappear. When this condition is fulfilled, one reaches situations where the quasi-classical image of the Anderson phase is not always sufficient to reproduce the quantum results.

Acknowledgments: Laboratoire Kastler Brossel is "UMR 8552 du CNRS, de l'ENS, et de l'Université Pierre et Marie Curie". 


\section{Appendix: angles of measurement}

To find the maximal violations of the Bell inequalities we used a numerical routine that produced the angles at which the violation occurred as well as the value of the violation. In the cases shown in Fig. 3 where $\mathcal{A}$ (and $\mathcal{B}, \mathcal{A}^{\prime}$, and $\mathcal{B}^{\prime}$ as well) is a product of results of measurements all at the same angle, we have noted previously that the angles at maximum occur in a fan arrangement with $b-a=a-b^{\prime}=a^{\prime}-b=\chi$, and $a^{\prime}-b^{\prime}=3 \chi$ (in this appendix we simplify the angle notation by writing, for example $\varphi_{a}=a$ ). In Fig. 4 we give $\chi$ for the same cases as treated in Fig. 3. For the case of $P=N / 2$ the angle $\chi$ drops off as $1 / \sqrt{N}$ as shown analytically with the Gaussian approximation of Eq. (39).

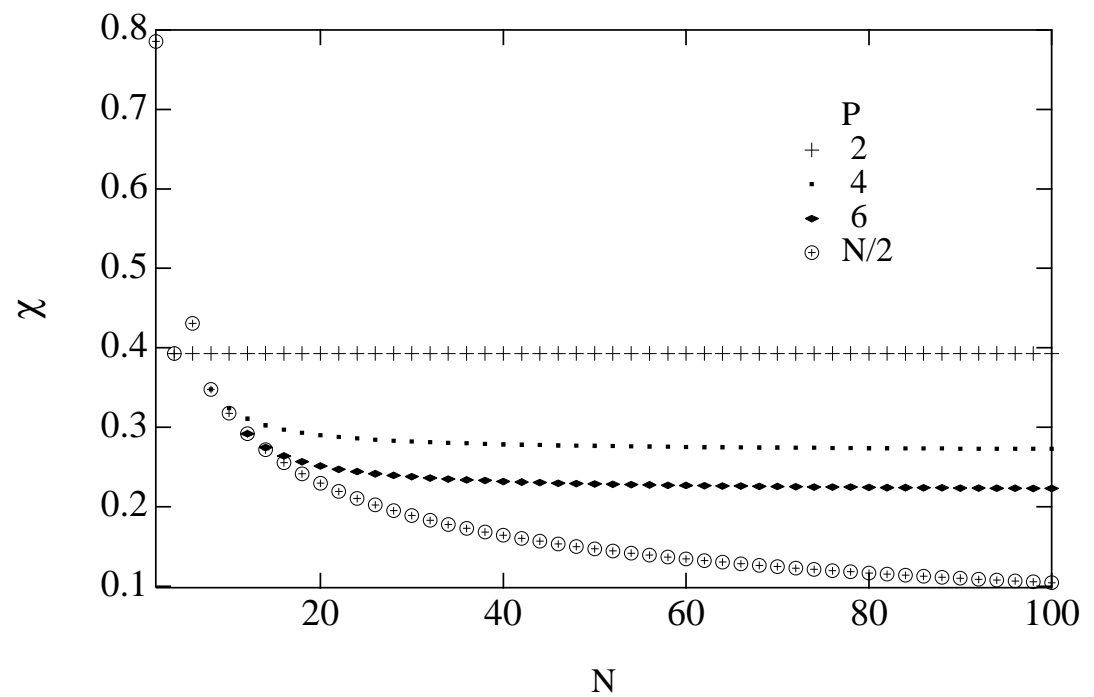

Figure 4: Angles of measurement corresponding to the violations shown in Fig. 3. The angle $\chi$ is defines the fan described in the text.

In the treatment of the "semi-mesoscopic" measurement described by Eq. (43), the angles also make a fan with $\chi=\pi / 4$ for the one case $N=6$ that gives a violation. However, when we analyze the inequality described in Eq. (44), the fan becomes distorted. We describe each of the situations of Table I individually. For $N=4$ all eight of the angles are distinct, but spread out in a pattern: Starting at $a^{\prime}$ and moving in order of $a^{\prime}, d^{\prime}, b, c, a, d, b^{\prime}, c^{\prime}$ we proceed alternately in steps of $\Delta_{1}$ and $\Delta_{2}$ where $\Delta_{1}=0.458$ and $\Delta_{2}=0.326$ (with $\Delta_{1}+\Delta_{2}=\pi / 4$ ). Thus the separation between any angle and its prime is $\pi / 2$. The pattern is shown in Fig. [5)(a).

For $N=6$, we use one measurement each for $\mathcal{A}$ and for $\mathcal{A}^{\prime}$, but the product of two measurements for $\mathcal{B}$ and for $\mathcal{B}^{\prime}$; similarly for $\mathcal{C}$ and $\mathcal{D}$ and their primes. The two angles for $\mathcal{B}$ collapse to a single angle with the same holding for $\mathcal{B}^{\prime}, \mathcal{D}$, and $\mathcal{D}^{\prime}$. We find $a=c$; if we take these as the origin, then we have $a^{\prime}$ at $-3 \Delta ; b$ and $d^{\prime}$ at $-\Delta ; b^{\prime}$ and $d$ at $\Delta ;$ and $c^{\prime}$ at $3 \Delta$ where $\Delta=\pi / 8$. The spread of the whole fan is then $\pi$. See Fig. 5 (b)

For $N=8$ and 12, the angles collapse to just four distinct values. For $N=8$ we have each letter $\mathcal{A}$, $\mathcal{B}, \mathcal{A}^{\prime}$, and $B^{\prime}$ being a product of two experimental results at the same angle. If $a^{\prime}=d^{\prime}$ is the origin, then we move up in steps of $\Delta_{1}, \Delta_{2}$, and $\Delta_{1}$ to $b=c, a=d$, and $b^{\prime}=c^{\prime}$, respectively, where $\Delta_{1}=0.4533$ and $\Delta_{2}=0.1738$. as seen in Fig. 5 (c). With $N=12$, each letter represents the product of three experimental 


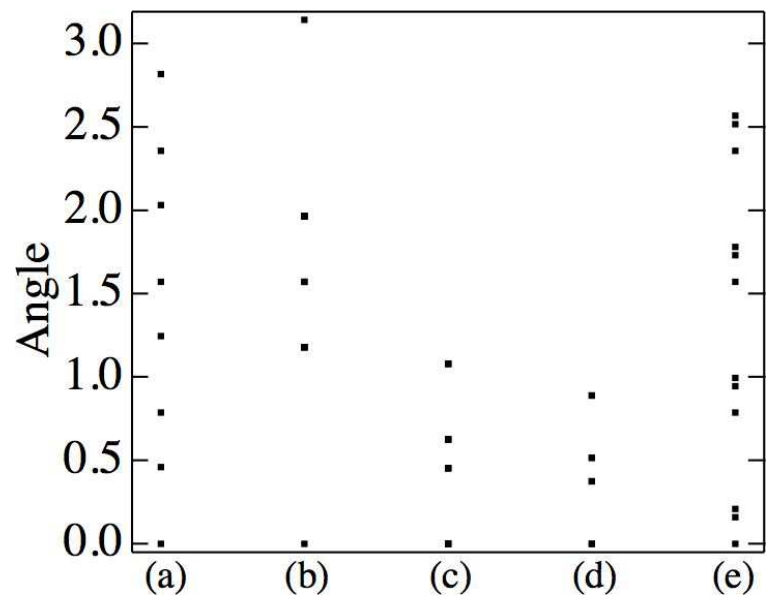

Figure 5: Angles of measurement corresponding to the inequalities of Eqs. (44) and (45). Patterns (a) through (d) show angles for $N=4,6,8$, and 12, respectively, corresponding to the violations of Table I for inequality (44). Part (e) shows the angle pattern for the violation of Eq. (45).

results at the same angle. The arrangement is the same as for $N=8$, except that the separations are reduced to $\Delta_{1}=0.3741$ and $\Delta_{2}=0.0685$, as shown in Fig. 5 (d). The Gaussian approximation shows the angle separations to be dropping as $1 / \sqrt{N}$.

Finally we have the inequality of Eq. (45). For $N=6$, each letter represents just one measurement. The angles resulting in a maximum are all distinct but come in four evenly spaced groups of three as seen in Fig. [5 (e). If the set $\left\{a^{\prime}, e^{\prime}, d^{\prime}\right\}$ is at $\{0,0.159,0.209\}$, then we move up by $\pi / 4$ to the next triplet of $\{b, f, c\} ; \pi / 4$ to the next set of $\{a, e, d\}$; and another $\pi / 4$ to $\left\{b^{\prime}, f^{\prime}, c^{\prime}\right\}$.

\section{References}

[1] A. Einstein, "Quantentheorie des einatomigen idealen Gases", Preussiche Akademie der Wissenchagten, Phys.-Math. Klasse, Sitzungsberichte, p. 3-14 (1925).

[2] A. Einstein, B. Podolsky and N. Rosen, "Can quantum mechanical description of physical reality be considered complete?", Phys. Rev. 47, 777-780 (1935).

[3] N. Bohr, "Can quantum mechanical description of physical reality be considered complete?", Phys. Rev. 48, 696-702 (1935)

[4] F. Laloë, "The hidden phase of Fock states, quantum non-local effects", Europ. Phys. J. D, 33, 87-97 (2005); "Bose Einstein condensates and quantum non-locality", page 35 in "Beyond the quantum", T.M. Nieuwenhuizen, V. Spicka, B. Mehmadi and A. Khrennikov editors, World Scientific (2007) and cond-mat/0611043

[5] J.S. Bell, "On the Einstein-Podolsky-Rosen paradox", Physics 1, 195-200 (1964); reprinted in [6].

[6] J.S. Bell, "Speakable and unspeakable in quantum mechanics", Cambridge University Press (1987).

[7] F. Laloë and W.J. Mullin, "Nonlocal quantum effects with Bose-Einstein condensates", Phys. Rev. Lett. 99, 150401 (2007).

[8] J.S. Bell, Comments on at. and mol. Phys. 9, 121 (1979); reprinted in 6]. 
[9] J. Javanainen and Sun Mi Yoo, "Quantum phase of a Bose-Einstein condensate with an arbitrary number of atoms", Phys. Rev. Lett. 76, 161-164 (1996).

[10] T. Wong, M.J. Collett and D.F. Walls, "Interference of two Bose-Einstein condensates with collisions", Phys. Rev. A 54, R3718-3721 (1996)

[11] J.I. Cirac, C.W. Gardiner, M. Naraschewski and P. Zoller, "Continuous observation of interference fringes from Bose condensates", Phys. Rev. A 54, R3714-3717 (1996).

[12] Y. Castin and J. Dalibard, "Relative phase of two Bose-Einstein condensates", Phys. Rev. A 55, 4330-4337 (1997)

[13] K. Mølmer, "Optical coherence: a convenient fiction", Phys. Rev. A 55, 3195-3203 (1997).

[14] Y. Castin and C. Herzog, "Bose-Einstein condensates in symmetry breaking states", C.R. Acad. Sci. série IV, 2, 419-443 (2001).

[15] C.J. Pethick and H. Smith, "Bose-Einstein condensation in dilute gases", Cambridge University Press (2002); see chap. 13.

[16] K. Mølmer, "Macroscopic quantum-state reduction: uniting Bose-Einstein condensates by interference measurements", Phys. Rev. A 65, 021607(R) (2002).

[17] P. Horak and S.M. Barnett, "Creation of coherence in Bose-Einstein condensates by atom detection", J. Phys. B 32, 3421-3436 (1999).

[18] M. Wheeler, K. Mertes, J. Erwin and D. Hall, "Spontaneous macroscopic spin polarization in independent spinor Bose-Einstein condensates", Phys. Rev. Lett. 93, 170402 (2004).

[19] J.F. Clauser, M.A. Horne, A. Shimony and R.A. Holt, "Proposed experiment to test local hiddenvariables theories", Phys. Rev. Lett. 23, 880-884 (1969).

[20] J.F. Clauser and A. Shimony, "Bell's theorem: experimental tests and implications", Rep. on Progress in Phys. 41, 1883-1926 (1978).

[21] A. Peres, "Unperformed experiments have no results", Am. J. Phys. 46, 745-47 (1978).

[22] B.S. Cirel'son, "Quantum generalizations of Bell's inequality", Letters in math. phys. 4, 93-100 (1980).

[23] A.J. Leggett and F. Sols, "On the concept of spontaneously broken gauge symmetry in condensed matter physics", Found. of Physics 21, 353-64 (1990)

[24] E. Siggia and A. Ruckenstein, "Bose condensation in spin-aligned atomic hydrogen", Phys. Rev. Lett. 44, 1423-1426 (1980).

[25] W.J. Mullin, R. Krotkov and F. Laloë, "Evolution of additional (hidden) quantum variables in the interference of Bose-Einstein condensates", Phys. Rev. A74, 023610 (2006).

[26] N.D. Mermin, "Extreme quantum entanglement in a superposition of macroscopically distinct states", Phys. Rev. Lett. 65, 1838 (1990).

[27] Zhi Zhao, Yu-Ao Chen, An-Ning Zhang, Tao Yang, Hans J. Briegel and Jian-Wei Pan, "Experimental demonstration of five-photon entanglement and open destination teleportation", Nature 430, 54 (1 July 2004). 
[28] J.A. Dunningham, K. Burnett and S.M. Barnett, "Interferometry below the standard quantum limit with Bose-Einstein condensates", Phys. Rev. Lett. 89, 150401 (2002).

[29] M.J. Holland and K. Burnett, "Interferometric detection of optical phase shifts at the Heisenberg limit", Phys. Rev. Lett. 71, 1355 (1993).

[30] P. Drummond, "Violations of Bell's inequality in cooperative states", Phys. Rev. Lett. 50, 1407 (1983).

[31] W. Dür, G. Vidal and J.I. Cirac, "Three qubits entangled in two inequivalent ways", Phys. Rev. A62, 062314 (2000)

[32] A. Cabello, "Bell's theorem with and without inequalities for the three-qubit GHZ and W states", Phys. Rev. A65, 032108 (2002); "Two qubits of a W state violate Bell's inequality beyond Cirel'son's bound", Phys. Rev. A66, 042114 (2002).

[33] A. Sen(De), U. Sen, M. Wiesniak, D. Kaszlikowski and M. Zukowski, "Family of ZeilingerHorne-Greenberger W states leads to stronger nonclassicality than family of GHZ states", arXiv: quant-ph/0211023v3 (2004).

[34] S. Ashhab and A.J. Leggett, "Bose-Einstein condensation of spin $1 / 2$ atoms with conserved total spin", Phys. Rev. A68, 063612 (2003). 